\title{
Modal-based prediction of sound transmission through slits and openings between rooms
}

\author{
J. Poblet-Puig;, A. Rodríguez-Ferran ${ }^{\dagger}$ \\ Laboratori de Càlcul Numèric \\ E.T.S. d'Enginyers de Camins, Canals i Ports de Barcelona \\ Universitat Politècnica de Catalunya
}

December 11, 2012

\begin{abstract}
The transmission of sound through slits and openings between cuboid-shaped rooms is analysed. A deterministic model that describes the pressure fields inside the rooms in terms of eigenfunctions and uses the Dirichlet-to-Neumann technique in order to reproduce the slit effect is presented. An efficient formulation of the problem is obtained thanks to the splitting of the original domain into three domains: sending room, slit, receiving room. The geometry and boundary conditions of the problem can be modelled in detail like in an element-based numerical technique (such as the finite element method) but with smaller computational costs. The model is compared with numerical solutions, existent models and published experimental data. Afterwards it is used to analyse some aspects such as the influence of slit dimensions, opening position, room properties (dimensions and absorption) that cannot be taken into account with the available models. These usually suppose that the slit or opening connects two unbounded acoustic domains.
\end{abstract}

\section{Introduction}

The sound transmission performance of walls is often worse than expected. The existence of slits and other small openings is one of the possible causes of this decrease in the insulation capacity. They are generated due to defective manufacturing or mountings. Sometimes the openings are a design requirement like the spaces in the surroundings of doors and windows, or in the installation of air conditioning systems.

*correspondence: UPC, Campus Nord B1, Jordi Girona 1, E-08034 Barcelona, Spain, e-mail: jordi.poblet@upc.edu

†e-mail: antonio.rodriguez-ferran@upc.edu 
In [18] it is shown how the slit under a door can completely modify its insulation capacity, specially in the high-frequency range where the insulation of the door (understood as a structure) is high while the sound that can be transmitted through the slit does not decrease. In $[12,13]$ airborne (structure path) and slit sound transmission models are applied in order to predict the insulation capacity of doors. This is the balance of two transmission paths: through the door itself and through the slits between the door and the surroundings (typically the floor). The laboratory measurements reveal quite good agreement with the models. One of the main conclusions of the work $[12,13]$ is that it is necessary to consider the slit path for a correct characterisation of the doors. The importance of the mounting procedures and quality control is illustrated by [17]. The differences in the insulation capacity of ship cabin walls measured in the ship (after installation) or in the laboratory can be explained by poor adjustments during the installation of the walls. Slits are also important in building lightweight constructions as shown in [32, 33].

A first group of models considers a baffled slit or opening between unbounded acoustic domains. A plane pressure wave excites the slit on the sending side. On this side, reflected and scattered waves are also generated. Inside the slit or hole, the pressure field is described by means of plane waves [35, 11, 10]. For this reason, these models can only be applied for small openings. In the same line a more detailed model is presented in [20]. The model improves the modelling of the three-dimensional effect of the rooms on the slit due to the excitation waves considered. In addition, the possibility of sealing the slits with some material is considered. All these models need to use expressions for the radiation impedances.

Other models try to enrich the pressure field inside the connecting element in order to deal with large openings. This group of models focuses its attention in the description of the pressure field inside the opening and how this is coupled with the surroundings. For the incident pressure wave and the radiated sound well known formulations are used. The pressure field inside the opening is described by means of trigonometric functions. A complete set is required in the transmission direction (typically sines and cosines are used) while the contours are supposed to be rigid (and then an interpolation of the pressure field by means of cosines is enough). Among them, it is worth mentioning the model presented in [4], where comparisons with laboratory experiments are carried out. In [24], the opening is rectangular and the equations are solved in the wavenumber domain after appropriate Fourier transforms. A similar model is proposed in [15] where an important part of the discussion is centred in the procedure used to improve the set of functions describing the pressure field inside the slit. Finally, in [28] a more general model that can take into account any opening shape is proposed. The presentation of the model is preceded by an extensive literature review and comparisons with experiments in [31].

Several hybrid models (numerical and statistical) are developed in [25]. Finite elements are used in order to describe the detail of holes in panels while statistical energy analysis is used in the other parts of the problem. Small holes with different cross-section shape are analysed. It is concluded that aspects such as the crosssection area or the hole length seem to be enough in order to perform predictions at low frequencies where the pressure field through the hole is one-dimensional.

The effect of finite dimension of rooms and walls have already been studied for the 
case of sound transmission through structures. In [3] two situations are compared. On the one hand panels placed in an infinite baffle, separating semi-infinite acoustic domains. On the other hand panels between finite dimension rooms. Results show differences that can be around $20 \mathrm{~dB}$ in the low-frequency range due to the modal response of the rooms. [19] study the effect of room dimensions, absorption (reverberation time of rooms) and the position of the source in the transmission of sound at low frequencies through a partition. This is considered as a pure mass (infinitely rigid wall). The pressure field in the rooms is solved by means of modal analysis and comparisons with finite elements and measurements are done. All results are compared with the mass-law showing discrepancies that can be around $\pm 10 \mathrm{~dB}$. There are other more complete models for sound transmission through walls based on modal analysis. [8] use a modal expansion for the cuboid rooms and for the rectangular single wall. They provide criteria to truncate the modal basis. [16] propose a decoupled approach where the sound transmission through isotropic and orthotropic plates with different boundary conditions is predicted. In [5] finite elements are used in order to make a more accurate description of the partition and consider a double wall. Layered partitions are also considered in [6] by means of wave-based and transfer matrix-based models that take into account modal coupling between the problem subsystems as well as corrections due to the finite dimensions of the problem.

The main contributions of this paper are:

- The development of a deterministic model that can be used in order to perform predictions of the sound transmitted through slits and openings connecting rooms of finite dimension. The available models known by the authors focus their attention on slits or openings that connect unbounded acoustic domains. The presented model lies in between i) simplified approaches with significant assumptions (unbounded domains, two-dimensional analysis, particular geometries) that lead to simple expressions of the sound transmission properties of the slit and ii) detailed approaches based on numerical techniques such as the finite (FEM) or boundary (BEM) element methods. On the one hand the geometry of the problem (room dimensions, position of the slit in the room wall) and the boundary conditions (acoustic absorption of the room, position of the sound source) can be taken into account like in the FEM or the BEM. Moreover, no hypotheses on the incident pressure field and the radiation efficiency of the slit are required. On the other hand pressure fields inside the rooms and the slit are described by means of eigenfunctions of uncoupled problems in order to reduce the computational burden. Regarding cost, the proposed model is closer to the simplified approaches than to the FEM or the BEM based models.

- Consequently, the presented model can be used in order to: i) verify the importance of considering the real dimensions of the rooms for this type of problems; ii) study the effect of parameters such as the acoustic absorption or the position of the slits and openings in the wall; iii) compare the results with finite element (or similar) based predictions. These can also be done with the FEM or the BEM but with higher computational costs and larger calculation times that often cause a reduction of the studied frequency range or the parametric analyses. 
- Parametric studies that can be used in order to perform acoustic designs involving slit leaks are provided.

- The calculations can be extended to higher frequencies as shown by the analysis of computational costs of Section 3.1 and the numerical results of the examples.

This introduction is followed by the formulation of the model in Section 2. Three different types of connecting elements are considered: holes, slits and openings. Numerical examples are presented afterwards in Section 3. Some of them are more related with the validation of the model and its implementation and some others are applications. The paper is closed with the conclusions in Section 4.

\section{Simplified model for the sound transmission be- tween cuboid-shaped rooms}

The formulation of a simplified model for the sound transmission through holes, slits and openings connecting cuboid-shaped rooms will be exposed here. The pressure field inside the rooms is described by means of the undamped eigenfunctions of the room. They have a well known expression [26, 34]. This is a good option if the connections between the rooms are weak and do not largely modify the modal pattern of the system. This is the case for holes, thin slits or small openings.

With this model the large costs of the resolution of the Helmholtz equation by means of the finite element method can be reduced. In addition, its implementation is simpler and faster, and a mesh is not required.

Three subsystems are considered, see Fig. 1: i) sending room (on the left); ii) receiving room (on the right) and iii) the connecting element between the rooms (hole, slit or opening). The relationship between the rooms and the connecting element is established by means of a Dirichlet-to-Neumann strategy [9]. The effect of the slit on both rooms is considered as an imposed velocity (Neumann boundary of the rooms). In a complementary way, the effect of the rooms on the slit is considered by means of an imposed pressure (Dirichlet boundary condition on the slit). The interpolation of the pressure field inside the slit is designed in order to satisfy this boundary condition.

\subsection{Pressure field inside rooms}

The Helmholtz equation with appropriate boundary conditions must be satisfied in both rooms

$$
\begin{aligned}
& \triangle p(\mathbf{x})+k^{2} p(\mathbf{x})=-\mathrm{i} \omega q \delta\left(\mathbf{x}_{q}, \mathbf{x}\right) \quad \text { in } \Omega_{a c} \\
& \nabla_{\mathbf{n}} p(\mathbf{x})=-\mathrm{i} \rho \omega v_{n} \quad \text { on } \Gamma_{N} \\
& \nabla_{\mathbf{n}} p(\mathbf{x})=-\mathrm{i} \rho \omega A p(\mathbf{x}) \quad \text { on } \Gamma_{A}
\end{aligned}
$$

Here, $\mathrm{i}=\sqrt{-1}$ is the imaginary unit, $\omega=2 \pi f$ is the pulsation of the problem, $v_{n}$ is the phasor of imposed velocity on Neumann boundaries $\Gamma_{N}, A$ is the admittance in absorbing (Robin) boundaries $\Gamma_{A}, q$ is the phasor of source strength amplitude of the sound source placed at $\mathbf{x}_{q}, \delta$ is the Dirac-delta and $\Omega_{a c}$ is the acoustic domain. $\rho$ and 


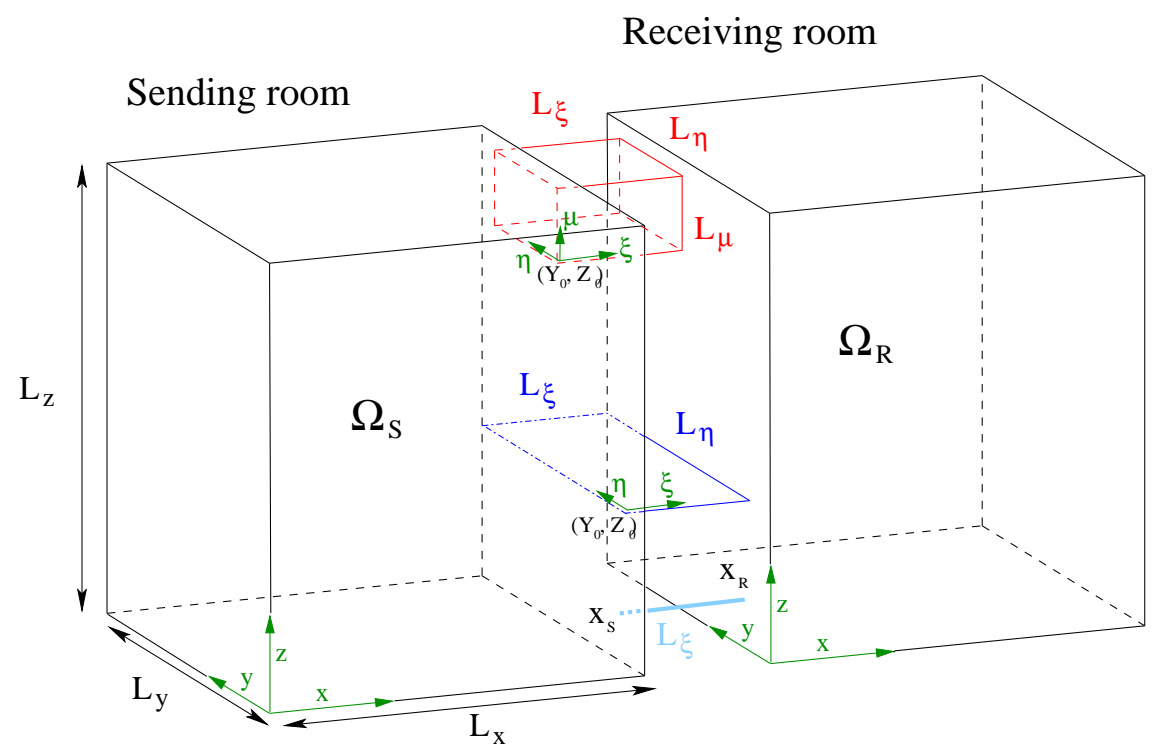

Figure 1: Two rooms connected through a hole (bottom), a slit (middle) and an opening (top).

$k=\omega / c$ are the density of the acoustic medium and the wavenumber respectively. In this work $\Gamma_{N}$ can be the interface between the room and a hole $\Gamma_{\text {hole }}$, a slit $\Gamma_{\text {slit }}$ or an opening $\Gamma_{\text {opening }}$.

The pressure fields are described by means of the undamped eigenmodes of each room

$$
p(\mathbf{x})=\sum_{j=1}^{n_{S}} a_{j} \psi_{j}(\mathbf{x}) \text { (sending) } \quad p(\mathbf{x})=\sum_{q=1}^{n_{R}} b_{q} \varphi_{q}(\mathbf{x}) \text { (receiving) }
$$

where $a_{j}$ is the contribution of the $j$ th eigenfunction $\psi_{j}(\mathbf{x})$ in the sending room, and $b_{q}$ is the contribution of the $q$ th eigenfunction $\varphi_{q}(\mathbf{x})$ in the receiving room. $n_{S}$ and $n_{R}$ are the number of modes in the sending and receiving rooms.

If the acoustic domains are cuboids, the eigenfunctions are

$$
\psi_{j}(x, y, z)=\cos \left(\frac{n_{x} \pi}{L_{x}} x\right) \cos \left(\frac{n_{y} \pi}{L_{y}} y\right) \cos \left(\frac{n_{z} \pi}{L_{z}} z\right) \quad n_{x}, n_{y}, n_{z}=0,1,2, \ldots
$$

and the eigenfrequencies are

$$
f_{j}=\frac{c k_{j}}{2 \pi} \quad \text { with } \quad k_{j}^{2}\left(n_{x}, n_{y}, n_{z}\right)=\left(\frac{n_{x} \pi}{L_{x}}\right)^{2}+\left(\frac{n_{y} \pi}{L_{y}}\right)^{2}+\left(\frac{n_{z} \pi}{L_{z}}\right)^{2}
$$

where $k_{j}\left(n_{x}, n_{y}, n_{z}\right)$ is the wavenumber of the eigenfrequency $j$, defined by the number of half-waves in each direction $n_{x}, n_{y}$ and $n_{z} . c$ is the speed of sound and $L_{x}, L_{y}, L_{z}$ are the dimensions of the cuboid. The eigenfunction $\varphi$ and its eigenfrequencies can be defined as in Eqs. (5) and (6) but using the parameters of the receiving room. Other options different from these normal modes are available in the literature. In [22] a set 
of eigenfunctions adapted in order to satisfy the absorbing (Robin) boundary conditions imposed on the walls by means of acoustic impedances is presented. Another example of enriched modal basis can be found in [36] where the classical functions are combined with the free field Green's function in order to improve the precision of the interpolation (specially around the point sound source) and reduce the number of functions to be used. Both approaches could also be considered in the developments exposed below in order to improve the pressure fields inside the rooms.

A weak form based on the modal analysis and that includes the boundary terms will be used. Including boundary terms is important in order to couple the rooms with the connecting element. This can be done by means of the second Green identity

$$
\int_{\Omega} p \triangle u d \Omega-\int_{\Omega} u \triangle p d \Omega+\int_{\partial \Omega} u \nabla_{\mathbf{n}} p d \Gamma-\int_{\partial \Omega} p \nabla_{\mathbf{n}} u d \Gamma=0
$$

Replacing Eq. (4) in Eq. (7), choosing $u=\psi_{i}$, considering $\triangle \psi_{i}+k_{i}^{2} \psi_{i}=0$ and using the boundary conditions of Eqs. (2) and (3) we can obtain a weak form for the sending room

$$
\begin{array}{r}
\sum_{j=1}^{n_{S}} a_{j}\left(k^{2}-k_{i}^{2}\right) \int_{\Omega_{S}} \psi_{i}(\mathbf{x}) \psi_{j}(\mathbf{x}) \mathrm{d} \Omega-\mathrm{i} \rho \omega \int_{\Gamma_{N}} v_{n} \psi_{i}(\mathbf{x}) \mathrm{d} \Gamma \\
+\sum_{j=1}^{n_{S}} a_{j}(-\mathrm{i} \rho \omega) \int_{\Gamma_{A}} A \psi_{i}(\mathbf{x}) \psi_{j}(\mathbf{x}) \mathrm{d} \Gamma=-\rho \mathrm{i} \omega q \int_{\Omega_{S}} \delta\left(\mathbf{x}_{q}, \mathbf{x}\right) \psi_{i}(\mathbf{x}) \mathrm{d} \Omega
\end{array}
$$

An analogous equation can be obtained for the receiving room.

Eq. (8) allows to consider the effect of the connecting element on the room by means of an imposed velocity. The acoustic absorption of the room can also be considered by means of the Robin boundary condition.

Three connecting elements will now be presented. The differences between them are the hypotheses on the pressure field inside according to the dimensions of the connecting element: point connection or hole, line connection or slit, surface connection or opening.

\subsection{Point connection or hole}

The connection between both cuboid-shaped rooms is supposed to be a straight hole in this section (see the bottom connection in Fig. 1). It is assumed that the pressure field $p(\xi)$ inside the hole is one-dimensional. This is true for small cross-section elements. The hole is characterised by its cross-section area $\mathrm{S}$ and its length $L_{\xi}$. This is a simple case that does not require the use of additional variables in order to describe the pressure field inside the connection. Moreover it illustrates well the basic idea of the method that will be used in the following sections to model more complex connection types. A relationship between the pressure and normal velocity at the edges of the straight hole can be established:

$$
\left[\begin{array}{c}
v_{n}(0) \\
v_{n}\left(L_{\xi}\right)
\end{array}\right]=\left[\begin{array}{ll}
A_{11} & A_{12} \\
A_{21} & A_{22}
\end{array}\right]\left[\begin{array}{c}
p(0) \\
p\left(L_{\xi}\right)
\end{array}\right]
$$


The admittance coefficients in Eq. (9) are obtained by means of the analytical solution of a one-dimensional Helmholtz equation:

$$
\begin{aligned}
& A_{11}=A_{22}=\frac{\mathrm{e}^{\mathrm{i} k L_{\xi}}+\mathrm{e}^{-\mathrm{i} k L_{\xi}}}{\left(\mathrm{e}^{-\mathrm{i} k L_{\xi}}-\mathrm{e}^{\mathrm{i} k L_{\xi}}\right)} \frac{k}{\rho \omega} \\
& A_{12}=A_{21}=\frac{-2}{\left(\mathrm{e}^{-\mathrm{i} k L_{\xi}}-\mathrm{e}^{\mathrm{i} k L_{\xi}}\right)} \frac{k}{\rho \omega}
\end{aligned}
$$

This can be done if the pressure field inside the connecting hole is considered one-dimensional and composed of plane waves which is the case of holes with small cross-section area. Note that $v_{n}(0), v_{n}\left(L_{\xi}\right), p(0)$ and $p\left(L_{\xi}\right)$ are scalar values.

For the case of the sending room we can express the coupling term due to the hole as

$$
\mathrm{i} \rho \omega \int_{\Gamma_{\text {hole }}}-v_{n} \psi_{i}(\mathbf{x}) \mathrm{d} \Gamma=\mathrm{i} \rho \omega \int_{\Gamma_{\text {hole }}} \psi_{i}(\mathbf{x})\left(A_{11} p(0)+A_{12} p\left(L_{\xi}\right)\right) \mathrm{d} \Gamma
$$

where $\Gamma_{\text {hole }}$ is the contact surface between the hole and the room and $v_{n}$ is the imposed normal velocity (taken as positive when pointing outwards the sending room). This can be expressed in terms of the hole pressures in the interface surfaces with the sending room $p(0)$ and the receiving room $p\left(L_{\xi}\right)$ thanks to the constitutive equation of the hole formulated in terms of an admittance matrix in Eq. (9).

$v_{n}(0)$ is the normal velocity in the hole connection with the sending room while $v_{n}\left(L_{\xi}\right)$ is the normal velocity in the hole connection with the receiving room. Note that since outward normal vectors are always considered (for the rooms and for the hole), the imposed normal velocities in the rooms are then

$$
v_{n}^{S}=-v_{n}(0) \text { and } v_{n}^{R}=-v_{n}\left(L_{\xi}\right)
$$

The same can be done with $p(0)$ and $p\left(L_{\xi}\right)$. They must be equal to the pressure values in the sending and receiving rooms in the hole contact:

$$
p(0)=p\left(\mathbf{x}_{S}\right) \quad \text { and } \quad p\left(L_{\xi}\right)=p\left(\mathbf{x}_{R}\right)
$$

$p\left(\mathbf{x}_{S}\right)$ and $p\left(\mathbf{x}_{R}\right)$ are expressed according to Eq. (4) and evaluated in the adequate position in the sending room $\left(\mathbf{x}_{S}\right)$ and in the receiving room $\left(\mathbf{x}_{R}\right)$.

Operating analogously for the receiving room, its coupling with the hole can be expressed as

$$
\mathrm{i} \rho \omega \int_{\Gamma_{\text {hole }}}-v_{n} \varphi_{r}(\mathbf{x}) \mathrm{d} \Gamma=\mathrm{i} \rho \omega \int_{\Gamma_{\text {hole }}} \varphi_{r}(\mathbf{x})\left(A_{21} p(0)+A_{22} p\left(L_{\xi}\right)\right) \mathrm{d} \Gamma
$$

A system of linear equations for the global problem (sending room - connection receiving room) is obtained,

$$
\left[\begin{array}{cc}
\mathbf{S}+\mathbf{C}_{S S} & \mathbf{C}_{S R} \\
\mathbf{C}_{R S} & \mathbf{R}+\mathbf{C}_{R R}
\end{array}\right]\left[\begin{array}{l}
\mathbf{a} \\
\mathbf{b}
\end{array}\right]=\left[\begin{array}{c}
\mathbf{f}_{S} \\
\mathbf{f}_{R}
\end{array}\right]
$$

where the modal matrix for the sending room can be expressed as

$$
(\mathbf{S})_{i j}=\delta_{i j}\left(k^{2}-k_{i}^{2}\right) \int_{\Omega_{S}} \psi_{i}(\mathbf{x}) \psi_{j}(\mathbf{x}) \mathrm{d} \Omega-\mathrm{i} \rho \omega \int_{\Gamma_{A}} A(\mathbf{x}) \psi_{i}(\mathbf{x}) \psi_{j}(\mathbf{x}) \mathrm{d} \Gamma
$$


For the eigenfunctions of a cuboid-shaped acoustic domain of Eq. (5) we have

$$
\int_{\Omega_{S}} \psi_{i}(\mathbf{x}) \psi_{i}(\mathbf{x}) \mathrm{d} \Omega=\frac{L_{x} L_{y} L_{z}}{\epsilon_{x} \epsilon_{y} \epsilon_{z}} \quad \text { with } \quad \epsilon_{q}= \begin{cases}1 & \text { if } n_{q}=0 \\ 2 & \text { if } n_{q} \neq 0\end{cases}
$$

Matrix $\mathbf{R}$ can be similarly obtained for the receiving room. $\mathbf{a}$ and $\mathbf{b}$ are the vectors of unknown modal contributions for the sending and receiving rooms. $\mathbf{f}_{S}$ and $\mathbf{f}_{R}$ are the modal force vectors. The coupling matrix due to the hole can be calculated as

$$
\left[\mathbf{C}_{S S}\right]_{i j}=\mathrm{i} \rho \omega \int_{\Gamma_{\text {hole }}} A_{11} \psi_{i}\left(\mathbf{x}_{S}\right) \psi_{j}\left(\mathbf{x}_{S}\right) \mathrm{d} \Gamma \quad i, j=0,1, \ldots, n_{S}
$$

Due to the point contact between the hole and the room and the one-dimensional description of the pressure field inside the hole (no variation at cross-section level), all variables are constant in $\Gamma_{\text {hole }}$ and Eq. (18) can be simplified as

$$
\left[\mathbf{C}_{S S}\right]_{i j}=\mathrm{i} \rho \omega \mathrm{S} A_{11} \psi_{i}\left(\mathbf{x}_{S}\right) \psi_{j}\left(\mathbf{x}_{S}\right) \quad i, j=0,1, \ldots, n_{S}
$$

where $S=\int_{\Gamma_{\text {hole }}} \mathrm{d} \Gamma$ is the hole area. The other coupling matrices are expressed as

$$
\begin{array}{rlr}
{\left[\mathbf{C}_{S R}\right]_{i q}=\mathrm{i} \rho \omega \mathrm{S} A_{12} \psi_{i}\left(\mathbf{x}_{S}\right) \varphi_{q}\left(\mathbf{x}_{R}\right)} & i=0,1, \ldots, n_{S} & q=0,1, \ldots, n_{R} \\
{\left[\mathbf{C}_{R S}\right]_{r j}=\mathrm{i} \rho \omega \mathrm{S} A_{21} \psi_{j}\left(\mathbf{x}_{S}\right) \varphi_{r}\left(\mathbf{x}_{R}\right)} & j=0,1, \ldots, n_{S} \quad r=0,1, \ldots, n_{R} \\
{\left[\mathbf{C}_{R R}\right]_{r q}=\mathrm{i} \rho \omega \mathrm{S} A_{22} \varphi_{r}\left(\mathbf{x}_{R}\right) \varphi_{q}\left(\mathbf{x}_{R}\right)} & r, q & =0,1, \ldots, n_{R}
\end{array}
$$

\subsection{Line connection or slit}

The idea presented in Section 2.2 has to be adapted when there is a slit (line) connecting the rooms. The slit can be understood as a thin connecting element where one of its dimensions (the width $h$ ) is much smaller than the other two (see the middle connection in Fig. 1). It will be assumed that the pressure inside the slit is twodimensional and varies with the local variables $\xi$ and $\eta$ but remains constant across the width. This is true for thin elements with a width $h$ that is much smaller than an acoustic wave length: $h \ll c / f$. With this hypothesis the pressure inside the slit can be interpolated as

$$
p(\xi, \eta)=\sum_{m=0}^{n_{\eta}} \cos \left(\frac{m \pi \eta}{L_{\eta}}\right) p_{m}(\xi)
$$

where $n_{\eta}+1$ is the number of functions considered along the direction $\eta \cdot p_{m}(\xi)$ is a function describing the variation of pressure along direction $\xi$ (from the sending room to the receiving room). This function must be able to describe a generic pressure field inside the slit. Moreover, non-null derivatives in the interfaces with the rooms are important in order to apply the Dirichlet-to-Neumann technique. A function that satisfies these requirements is

$$
p_{m}(\xi)=\frac{1}{\gamma_{m}}\left(p_{m}^{S}\left(\mathrm{e}^{-\mathrm{i} k_{m} L_{\xi}} \mathrm{e}^{\mathrm{i} k_{m} \xi}-\mathrm{e}^{\mathrm{i} k_{m} L_{\xi}} \mathrm{e}^{-\mathrm{i} k_{m} \xi}\right)+p_{m}^{R}\left(\mathrm{e}^{-\mathrm{i} k_{m} \xi}-\mathrm{e}^{\mathrm{i} k_{m} \xi}\right)\right)
$$


where $p_{m}^{S}=p_{m}(\xi=0), p_{m}^{R}=p_{m}\left(\xi=L_{\xi}\right)$ and

$$
\gamma_{m}=\mathrm{e}^{-\mathrm{i} k_{m} L_{\xi}}-\mathrm{e}^{\mathrm{i} k_{m} L_{\xi}} \quad \text { with } \quad k_{m}=\sqrt{\left(\frac{\omega}{c}\right)^{2}-\left(\frac{m \pi}{L_{\eta}}\right)^{2}}
$$

The pressure field inside the slit shown in Eq. (21) satisfies the Helmholtz equation with wavenumber $k=\omega / c$ and the boundary conditions

$$
\begin{array}{lr}
\frac{\partial p}{\partial n}=0 & \text { on } \eta=0 \text { and } \eta=L_{\eta} \\
p(\xi=0, \eta)=\sum_{m=0}^{n_{\eta}} \cos \left(\frac{m \pi \eta}{L_{\eta}}\right) p_{m}^{S} & \text { on } \xi=0 \\
p\left(\xi=L_{\xi}, \eta\right)=\sum_{m=0}^{n_{\eta}} \cos \left(\frac{m \pi \eta}{L_{\eta}}\right) p_{m}^{R} & \text { on } \xi=L_{\xi}
\end{array}
$$

where $\partial p / \partial n$ is the normal derivative of the pressure field. The normal velocity at $\xi=0$ and $\xi=L_{\xi}$ can be expressed as

$$
\left[\begin{array}{c}
v_{n}(\xi=0, \eta) \\
v_{n}\left(\xi=L_{\xi}, \eta\right)
\end{array}\right]=\sum_{m=0}^{n_{\eta}} \cos \left(\frac{m \pi \eta}{L_{\eta}}\right)\left[\begin{array}{cc}
A_{11}^{m} & A_{12}^{m} \\
A_{21}^{m} & A_{22}^{m}
\end{array}\right]\left[\begin{array}{c}
p_{m}^{S} \\
p_{m}^{R}
\end{array}\right]
$$

where $A_{i j}^{m}$ is defined like in Eq. (10) with the appropriate value of $k=k_{m}$.

In a similar way to Eq. (11) the coupling of the slit with the sending room $(\xi=0$, $x=L_{x}$ ) can be modelled as an imposed velocity from the slit to the room:

$$
\mathrm{i} \rho \omega \int_{\Gamma_{\text {slit }}}-v_{n} \psi_{i}(\mathbf{x}) \mathrm{d} \Gamma=\mathrm{i} \rho \omega \sum_{m=0}^{n_{\eta}} \int_{\Gamma_{\text {slit }}} \cos \left(\frac{m \pi \eta}{L_{\eta}}\right)\left(A_{11}^{m} p_{m}^{S}+A_{12}^{m} p_{m}^{R}\right) \psi_{i}(\mathbf{x}) \mathrm{d} \Gamma
$$

The same can be done with the receiving room $\left(\xi=L_{\xi}, x=0\right)$ :

$$
\mathrm{i} \rho \omega \int_{\Gamma_{\text {slit }}}-v_{n} \varphi_{i}(\mathbf{x}) \mathrm{d} \Gamma=\mathrm{i} \rho \omega \sum_{m=0}^{n_{\eta}} \int_{\Gamma_{\text {slit }}} \cos \left(\frac{m \pi \eta}{L_{\eta}}\right)\left(A_{21}^{m} p_{m}^{S}+A_{22}^{m} p_{m}^{R}\right) \varphi_{i}(\mathbf{x}) \mathrm{d} \Gamma
$$

The coupling is completed by imposing the continuity of pressure between the slit and the cuboid rooms. In the sending room we have

$$
\begin{aligned}
& \sum_{m=0}^{n_{\eta}} \cos \left(\frac{m \pi \eta}{L_{\eta}}\right) p(\xi=0, \eta)=\sum_{j=1}^{n_{S}} a_{j} \psi_{j}\left(x=L_{x}, y=y(\eta), z=z(\eta)\right) \\
& \forall \eta \in\left[0, L_{\eta}\right]
\end{aligned}
$$

which can be imposed in weak form as

$$
\begin{array}{r}
\sum_{m=0}^{n_{\eta}} p_{m}^{S} \int_{\Gamma_{\text {slit }}} \cos \left(\frac{n \pi \eta}{L_{\eta}}\right) \cos \left(\frac{m \pi \eta}{L_{\eta}}\right) \mathrm{d} \Gamma=\sum_{j=1}^{n_{S}} a_{j} \int_{\Gamma_{\mathrm{slit}}} \cos \left(\frac{n \pi \eta}{L_{\eta}}\right) \psi_{j} \mathrm{~d} \Gamma \\
n=0,1, \ldots, n_{\eta}
\end{array}
$$


And analogously for the receiving room:

$$
\begin{array}{r}
\sum_{m=0}^{n_{\eta}} p_{m}^{R} \int_{\Gamma_{\text {slit }}} \cos \left(\frac{n \pi \eta}{L_{\eta}}\right) \cos \left(\frac{m \pi \eta}{L_{\eta}}\right) \mathrm{d} \Gamma=\sum_{j=1}^{n_{R}} b_{j} \int_{\Gamma_{\text {slit }}} \cos \left(\frac{n \pi \eta}{L_{\eta}}\right) \varphi_{j} \mathrm{~d} \Gamma \\
n=0,1, \ldots, n_{\eta}
\end{array}
$$

Finally, the linear systems of equations to be solved is

$$
\left[\begin{array}{cccc}
\mathbf{S} & \mathbf{0} & \mathbf{C}_{S, P s} & \mathbf{C}_{S, P r} \\
\mathbf{0} & \mathbf{R} & \mathbf{C}_{R, P s} & \mathbf{C}_{R, P r} \\
\mathbf{C}_{P s, S} & \mathbf{0} & \mathbf{D} & \mathbf{0} \\
\mathbf{0} & \mathbf{C}_{P r, R} & \mathbf{0} & \mathbf{D}
\end{array}\right]\left[\begin{array}{c}
\mathbf{a} \\
\mathbf{b} \\
\mathbf{p}_{S} \\
\mathbf{p}_{R}
\end{array}\right]=\left[\begin{array}{c}
\mathbf{f}_{S} \\
\mathbf{f}_{R} \\
\mathbf{0} \\
\mathbf{0}
\end{array}\right]
$$

where $\mathbf{S}, \mathbf{R}, \mathbf{a}, \mathbf{b}, \mathbf{f}_{S}$ and $\mathbf{f}_{R}$ have been defined after Eq. (15) and

$$
[\mathbf{D}]_{n m}=\int_{\eta=0}^{L_{\eta}} \cos \left(\frac{n \pi \eta}{L_{\eta}}\right) \cos \left(\frac{m \pi \eta}{L_{\eta}}\right) \mathrm{d} \eta \quad n, m=0,1,2, \ldots, n_{\eta}
$$

$\mathbf{p}_{S}$ and $\mathbf{p}_{R}$ are vectors including the unknown scalars $p_{m}^{S}$ and $p_{m}^{R}$ respectively.

In order to compute the coupling matrices $\mathbf{C}$ between the rooms and the slit, the following integrals are needed:

$$
\begin{aligned}
\beta_{i m} & =\int_{\Gamma_{\text {slit }}} \cos \left(\frac{m \pi \eta}{L_{\eta}}\right) \psi_{i}(\mathbf{x}) \mathrm{d} \Gamma \quad i=0,1, \ldots, n_{S} \text { and } m=1,2, \ldots, n_{\eta} \\
\kappa_{i m} & =\int_{\Gamma_{\text {slit }}} \cos \left(\frac{m \pi \eta}{L_{\eta}}\right) \varphi_{i}(\mathbf{x}) \mathrm{d} \Gamma \quad i=0,1, \ldots, n_{R} \text { and } m=1,2, \ldots, n_{\eta}
\end{aligned}
$$

The slit surface in contact with the sending and receiving rooms $\left(\Gamma_{\text {slit }}\right)$ can be considered a line ( $\eta$ direction) of width $h$ and the integrals in Eq. (35) can be simplified:

$$
\begin{gathered}
\beta_{i m}=h \int_{\eta=0}^{L_{\eta}} \cos \left(\frac{m \pi \eta}{L_{\eta}}\right) \psi_{i}\left(x=L_{x}, y=y(\eta), z=z(\eta)\right) \mathrm{d} \eta \\
\kappa_{i m}=h \int_{\eta=0}^{L_{\eta}} \cos \left(\frac{m \pi \eta}{L_{\eta}}\right) \varphi_{i}(x=0, y=y(\eta), z=z(\eta)) \mathrm{d} \eta
\end{gathered}
$$

They do not depend on the frequency but only on the geometry (slit shape and the relative position between the slit and the rooms). For this reason they can be precalculated before a frequency loop. It is very useful in order to compute all the coupling matrices involved in the problem:

$$
\begin{aligned}
{\left[\mathbf{C}_{P s, S}\right]_{m i}=-\beta_{i m} } & {\left[\mathbf{C}_{P r, R}\right]_{m i}=-\kappa_{i m} } \\
{\left[\mathbf{C}_{S, P s}\right]_{i m}=\mathrm{i} \rho \omega A_{11}^{m} \beta_{i m} } & {\left[\mathbf{C}_{S, P r}\right]_{i m}=\mathrm{i} \rho \omega A_{12}^{m} \beta_{i m} } \\
{\left[\mathbf{C}_{R, P s}\right]_{i m}=\mathrm{i} \rho \omega A_{21}^{m} \kappa_{i m} } & {\left[\mathbf{C}_{R, P r}\right]_{i m}=\mathrm{i} \rho \omega A_{22}^{m} \kappa_{i m} }
\end{aligned}
$$

Planar slits are considered here. However, the formulation could be extended to thin curve-shaped slits in the wall plane extruded along the $\xi$ direction. Eq. (21) remains still valid but considering that $\eta$ is a coordinate following the medial axis of the slit and $L_{\eta}$ is the length of this medial axis. The major difficulty would be to calculate the integrals in Eq. (35). This could be done numerically. 


\subsection{Surface connection or opening}

The idea presented in Section 2.3 can be directly extended to surface connections or openings where the variation of pressure along no direction can be neglected and the pressure field inside the connection is three-dimensional. This could be the case of a window connecting two rooms or an air conditioning tube of middle dimensions. See the top connection of Fig. 1. The presented approach must be limited to openings whose dimension is small when compared to the room wall. If the opening is large, this modifies very much the modal pattern of the system. An extreme situation is reached when the dimensions of the opening and the room wall are the same. Thus, we have one cuboid shaped room and the modal pattern of this problem largely differs from the modal pattern that can be reproduced with the three subsystems (composed of eigenmodes of each isolated room and the connecting element). The effect of openings on the eigenmodes of a two-dimensional room has been studied in [29].

The main modification to be done is to substitute the one-variable function $\cos \left(\frac{m \pi \eta}{L_{\eta}}\right)$ by a two-variable function $\chi(\eta, \mu)$. All the integrals over $\Gamma_{\text {slit }}$, see Eq. (35), which are reduced to one-dimensional integrals, see Eq. (36), have to be computed now over a two-dimensional surface $\Gamma_{\text {opening }}$ (contact surface between the openings and the rooms). The natural option for the rectangular opening of Fig. 1 is to choose an interpolation function in the $\eta-\mu$ plane such as

$$
\chi(\eta, \mu)=\cos \left(\frac{m \pi \eta}{L_{\eta}}\right) \cos \left(\frac{n \pi \mu}{L_{\mu}}\right)
$$

This considerably simplifies the operations to be done and maintains the orthogonality properties. For other opening shapes a generic set of functions $\chi(\eta, \mu)$ could be used. If they are $\eta-\mu$ plane eigenfunctions the same algorithm lay-out and matrix structure presented in Section 2.3 is kept. The function in Eq. (22) can still be used in order to deal with the $\xi$ direction.

The systems of linear equations to be solved are like in Eq. (33) but larger due to the larger number of modes to be considered in the $\eta-\mu$ plane. In both cases the system matrix is composed by blocks. The diagonal blocks are diagonal matrices and the off-diagonal blocks are full matrices. This can be exploited by a block Gauss-Seidel strategy as proposed in [27]. It is very fast to make an iteration of the method because it only requires to solve diagonal systems of equations and to compute matrix-vector products.

\subsection{Post-processing and outputs of interest}

The pressure level in the sending $\left(L_{S}\right)$ and receiving $\left(L_{R}\right)$ rooms can be calculated as

$$
L=10 \log _{10}\left(\frac{\left\langle p_{\mathrm{rms}}^{2}\right\rangle}{p_{0}^{2}}\right) \quad \text { with }\left\langle p_{\mathrm{rms}}^{2}\right\rangle=\frac{1}{n} \sum_{i=1}^{n} \frac{\left|p_{i}\right|^{2}}{2}
$$

where $\left\langle p_{\text {rms }}^{2}\right\rangle$ is the space averaged root mean square pressure (calculated by means of a discrete average of the pressure in $n$ random points). An alternative way to perform 
the spatial average is mode by mode (instead of random points). If the modes are uncoupled

$$
\left\langle p_{\text {rms }}^{2}\right\rangle=\frac{\int_{\Omega} \frac{|p(\mathbf{x})|^{2}}{2} \mathrm{~d} \Omega}{\int_{\Omega} \mathrm{d} \Omega}=\sum_{j=1}^{n_{S}} \frac{\left|a_{j}\right|^{2}}{2 L_{x} L_{y} L_{z}} \int_{\Omega}\left|\psi_{j}(\mathbf{x})\right|^{2} \mathrm{~d} \Omega
$$

Another aspect to take into account in order to optimise the computational costs is to properly choose the room modes. The option implemented here is to consider all the modes with eigenfrequency in $(f-\Delta f, f+\Delta f)$ and the ten lowest modes. In the studied examples a value of $\Delta f=100 \mathrm{~Hz}$ is used. Fig. 2 shows the percentage of modal contribution in the sum of Eq. (42) that is neglected due to the truncation. A value of $\Delta f=100 \mathrm{~Hz}$ implies that around $1 \%$ of the energy is neglected. This amount of energy neglected depends on $\Delta f$ but also on the acoustic absorption (for a constant error a larger modal base must be used if the acoustic absorption is larger). Fig. 3 shows the relative error in the sound reduction index for which the coupling between modes is important. Relative errors for the sound reduction index are smaller than for the energy. However this decrease is partially caused by the fact that the sound reduction index is a logarithmic output.

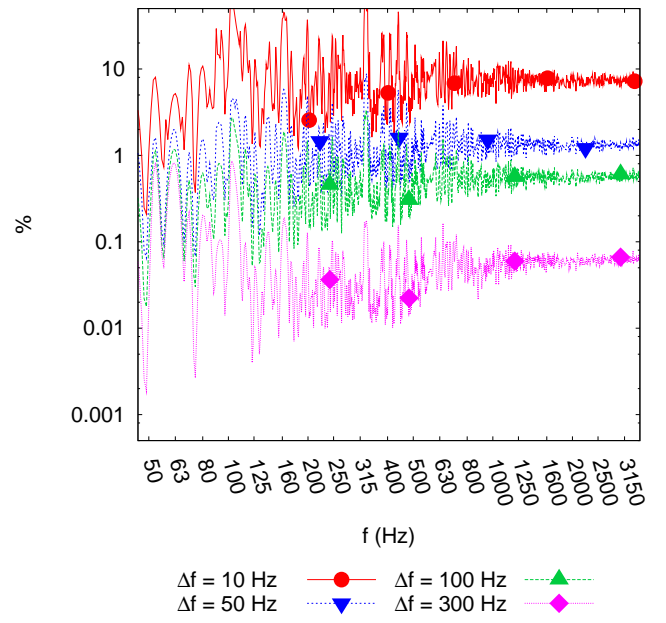

(a)

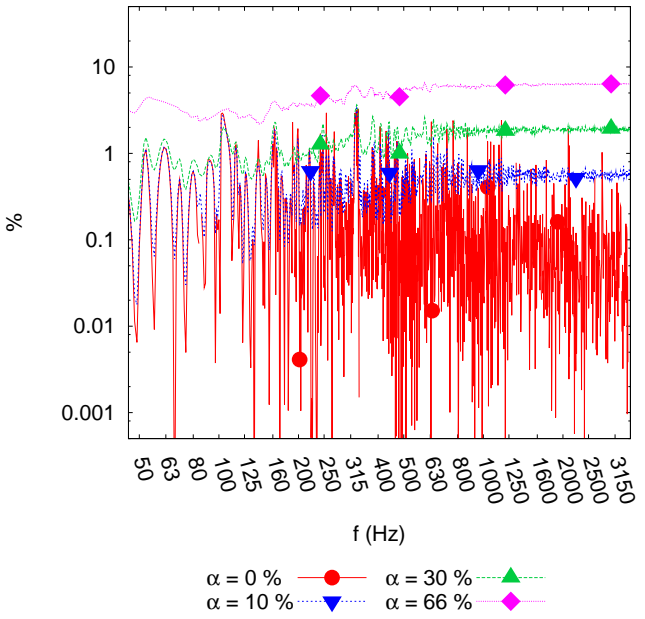

(b)

Figure 2: Influence of the number of modes used. Percentage of energy of the receiving room that is outside a frequency band $\left(\alpha=10 \%, L_{\xi}=0.05 \mathrm{~m}, h=0.05 \mathrm{~m}\right)$ : (a) Influence of bandwidth $\Delta f$; (b) Influence of room absorption for a fixed $\Delta f=100$ $\mathrm{Hz}$.

In more general situations of rooms connected by large openings, vibrating structures coupled to the cavity or rooms with very large absorption, there would be a strong modal coupling. In that cases, all the modes up to a given modal truncation frequency should be kept in the expansion. This truncation frequency can vary around 1.2 or 2 times the calculation frequency. The modal expansion could also be improved using pseudo-static corrections, see for example [30].

Most of the results in the following sections are presented in one-third octave frequency band averages. This is done by considering values of $\left\langle p_{\mathrm{rms}}^{2}\right\rangle$ at some frequencies 


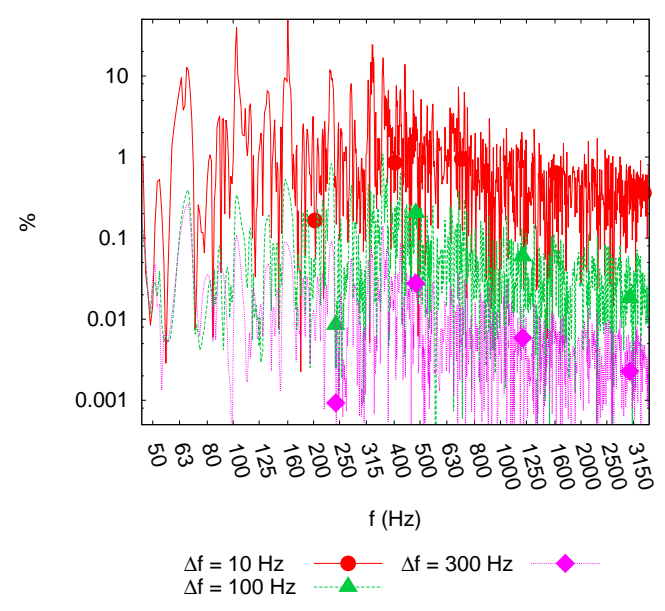

(a)

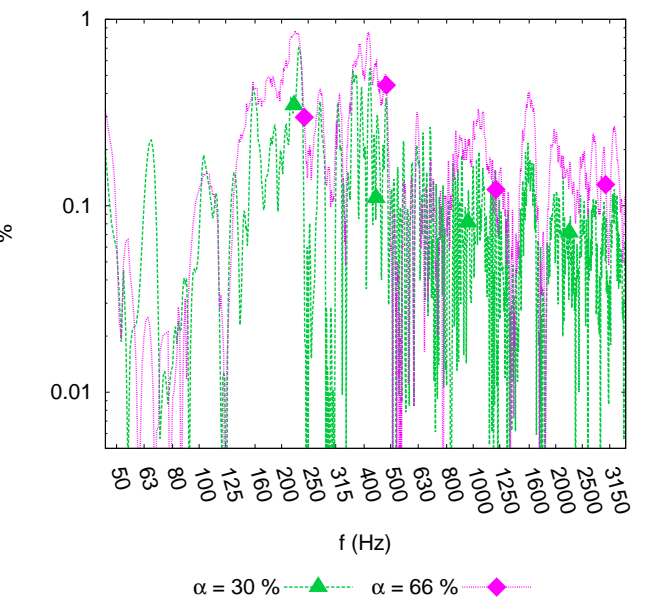

(b)

Figure 3: Influence of the number of modes used. Relative error in the sound reduction index $\left(L_{\xi}=0.05 \mathrm{~m}, h=0.05 \mathrm{~m}\right)$ : (a) Influence of bandwidth $\Delta f$ for constant $\alpha=10 \%$, ; (b) Influence of room absorption for a fixed $\Delta f=100 \mathrm{~Hz}$.

and then performing its frequency average

$$
\left\langle p_{\mathrm{rms}}^{2}\right\rangle_{\text {Band }}=\frac{\int_{f_{\mathrm{down}}}^{f_{\mathrm{up}}}\left\langle p_{\mathrm{rms}}^{2}\right\rangle(f) d f}{f_{\mathrm{up}}-f_{\mathrm{down}}}
$$

where $f_{\text {up }}$ and $f_{\text {down }}$ are the limit frequencies in the band. The integrals are performed numerically by means of a composite trapezoidal rule. It is also possible to use overall levels which is the output often measured in laboratory. However, this is equivalent in terms of sound reduction index predictions.

\section{$3 \quad$ Examples}

The performance of the method and its application to the acoustic design of slits and openings is shown here by means of several examples. In the first group the proposed model is first applied to a one-dimensional problem in order to verify the applicability of the Dirichlet-to-Neumann technique. Afterwards, a more complex two-dimensional problem with a hole connecting two rooms is solved by means of the proposed modal model and with the FEM, verifying that equivalent solutions are obtained. Finally, a comparison with the simplified model proposed in [10] as well as the laboratory measurements reported in [11] is done.

The application analyses mainly focus their attention on aspects that cannot be treated with most of the available models. Among them: the influence of the slit length (it can be analysed with the model presented in [28]), the effect of room properties (size and absorption) in this type of measurements or the relevance of the opening position. 
In all the examples shown here $c=340 \mathrm{~m} / \mathrm{s}$ and $\rho=1.18 \mathrm{~kg} / \mathrm{m}^{3}$. The real values of admittance $A$ for the absorbing (Robin) boundaries are taken from Table 1 where the random incidence absorption coefficient $\alpha$ calculated as proposed in $[1,21,2]$ is also shown (the average is three-dimensional and considering uniform distribution of angles for the incidence waves).

$$
\begin{gathered}
\alpha=2 \int_{0}^{\frac{\pi}{2}} \alpha(\theta) \sin (\theta) \cos (\theta) d \theta \quad \text { with } \alpha(\theta)=1-\left|\frac{\cos (\theta)-A \rho c}{\cos (\theta)+A \rho c}\right| \\
\begin{array}{ccccc}
1 / A \rho c & 6 & 20 & 32.5 & 70 \\
\hline \alpha(\%) & 66 & 30 & 20 & 10
\end{array}
\end{gathered}
$$

Table 1: Values of normalised admittance and random incidence absorption coefficient for the Robin boundary condition.

The value of $\alpha$ is frequency-dependent for real materials and in rooms with furniture. However, for the parametric analyses that are shown here, it seems more adequate to consider constant values of absorption. The formulation of the model does not change if a frequency-dependent value of $\alpha$ is used.

\subsection{Comparison with the FEM}

The problem sketched in Fig. 4 is solved here in two different ways: i) considering the two rooms and the connection as a single acoustic domain; and ii) partitioning the problem in three sub-domains and then applying the modal model described in Section 2.2. An analytical solution of the problem is not available. For this reason the monolithic problem is solved by means of a two-dimensional FEM analysis. The two solutions (monolithic using FEM and partitioned using the modal model) are compared here.

The dimensions of the sending room are $L_{x}=2 \mathrm{~m}$ and $L_{y}=3 \mathrm{~m}$, and for the receiving room $L_{x}=2.5 \mathrm{~m}$ and $L_{y}=3 \mathrm{~m}$. A hole of surface per unit length $\mathrm{S}=$ $5 \mathrm{~cm}^{2} / \mathrm{cm}$ and $L_{\xi}=0.1 \mathrm{~m}$ placed in the middle of the wall $\left(y_{\text {slit }}=1.5 \mathrm{~m}\right)$ connects both rooms. Values of absorption according to Table 1 are considered. A point sound source (with a source strength $q_{s}=1.5 \mathrm{~m}^{3} / \mathrm{s}$ ) is placed at position $X_{s}=0.49 \mathrm{~m}$, $Y_{s}=0.48 \mathrm{~m}$ in the sending room.

The pressure levels in the sending and receiving rooms are shown in Fig. 5. The agreement between the results obtained with the monolithic option using FEM and the partitioned approach that allows the use of the modal model is very good. This comment is also valid in the low-frequency range and showing outputs non-averaged in a frequency band.

Solution methods for acoustic problems based on the eigenfunctions of separate subdomains often provide acceptable results at mid/high frequencies and after averaging several frequencies. This second aspect is related with the importance of reproducing an equivalent modal density of the subsystems inside a frequency band (and it can be accepted to find a certain phase lag of results when looking at individual frequencies, a phenomenon that also happens with the FEM). These reasons explain 


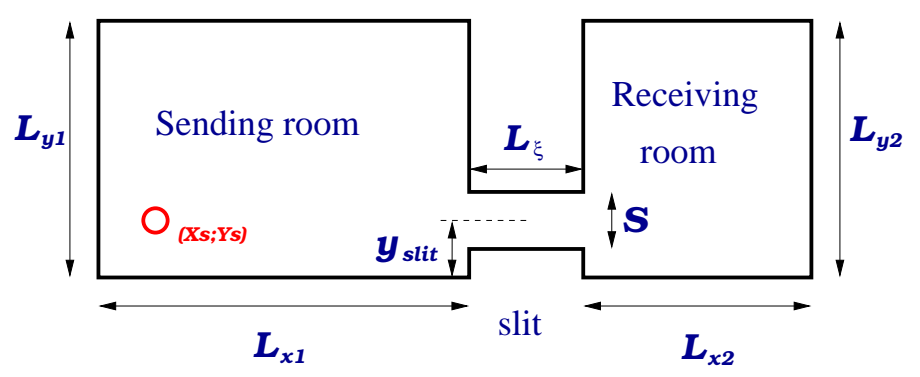

Figure 4: Sketch of a two-dimensional problem: two rooms connected through a hole.

the differences found in Fig. 5 between both approaches. However, the differences are small and this validates the quality of the predictions done by means of the modal model.

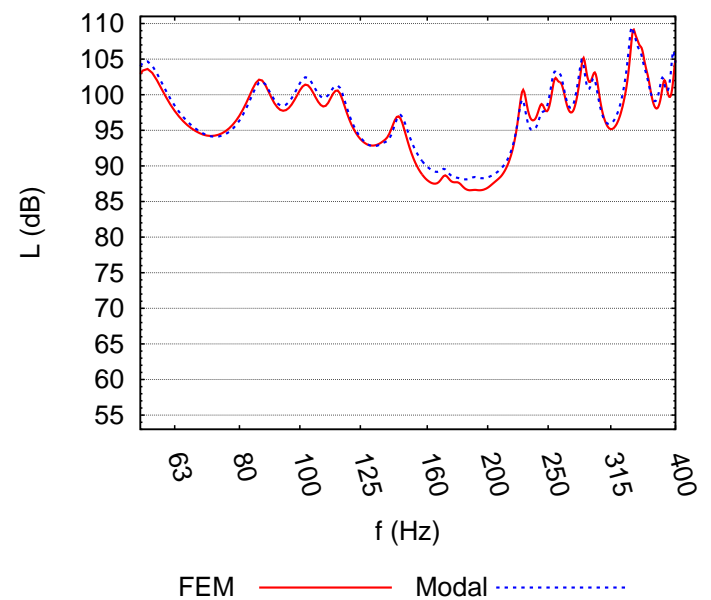

(a)

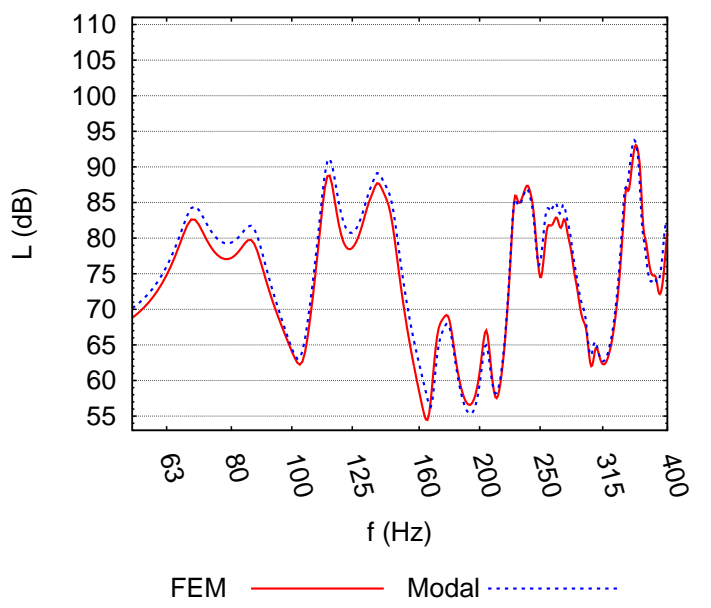

(b)

Figure 5: Comparison between the finite element method and the modal model $(\alpha=$ $30 \%, L_{\xi}=0.05 \mathrm{~m}, h=0.05 \mathrm{~m}$ ): (a) sending room; (b) receiving room.

The reduction of computational burden of the modal model with respect to a FEM model is moderate for two-dimensional problems. However, this is essential for threedimensional analyses. Three-dimensional FEM with the usual room dimensions can only be used for very low frequencies.

An estimation of the computational costs of both techniques for a typical threedimensional problem is done. The problem considered is the sound transmission between two cuboid-shaped rooms connected through a horizontal slit. The dimensions of the sending room are $L_{x}=2 \mathrm{~m}, L_{y}=3.5 \mathrm{~m}$ and $L_{z}=3 \mathrm{~m}$, and for the receiving room $L_{x}=2.5 \mathrm{~m}, L_{y}=3.5 \mathrm{~m}$ and $L_{z}=3 \mathrm{~m}$.

The two methods are very different and their cost will depend on several aspects such as the implementation or the type of matrices but this analysis can provide an approximated order of magnitude.

Fig. 6(a) shows the number of degrees of freedom depending on the frequency for 
each technique. In the FEM, the number of nodes $\left(n_{\mathrm{FEM}}\right)$ in each acoustic domain is calculated by supposing that six elements per acoustic wave length are used. Then, for a cuboid-shaped room we have:

$$
n_{\mathrm{FEM}} \simeq \frac{6^{3} L_{x} L_{y} L_{z}}{\lambda^{3}}
$$

where $\lambda=2 \pi / k$.

The number of acoustic modes in a cuboid-shaped acoustic domain below a frequency $f$ can be estimated as (see for example, [26])

$$
n_{\text {mod }}(k)=\frac{1}{6} \frac{L_{x} L_{y} L_{z}}{2 \pi} k^{3}+\frac{1}{4} \frac{L_{x} L_{y}+L_{x} L_{z}+L_{y} L_{z}}{2 \pi} k^{2}+\frac{1}{8} \frac{L_{x}+L_{y}+L_{z}}{\pi} k+\frac{1}{8}
$$

Eq. (46) can be used in order to calculate the number of eigenfunctions inside a band.

For the example studied here we can see that the proposed modal model drastically reduces the number of degrees of freedom. This is often the case of numerical techniques that use eigenfunctions as interpolation functions instead of the element approach of the FEM. This is specially important in order to deal with high frequencies.

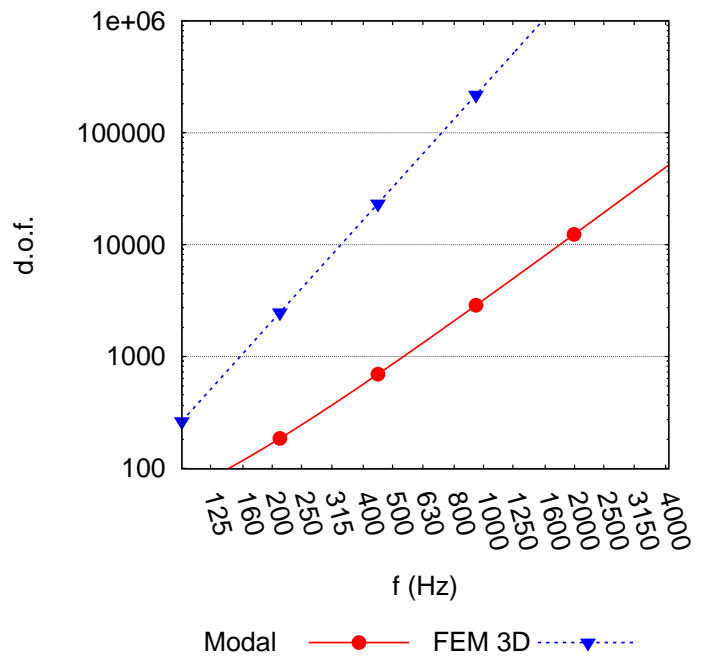

(a)

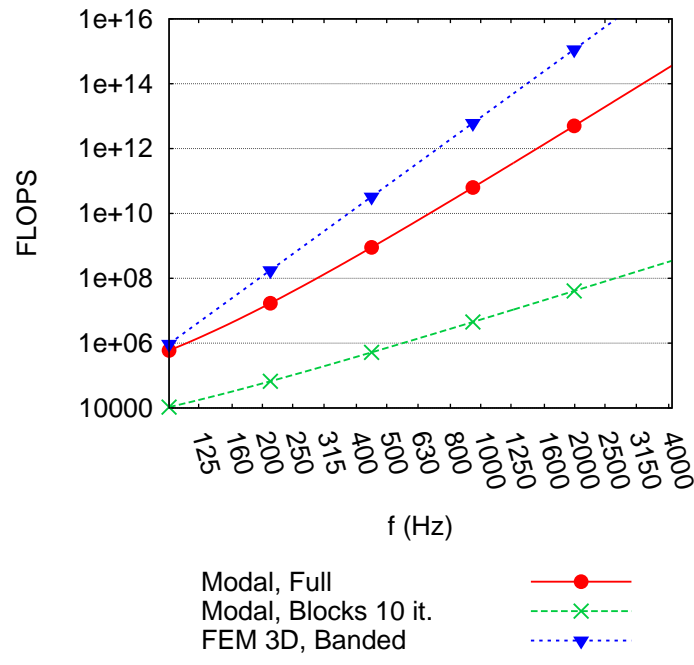

(b)

Figure 6: Approximation of the computational costs depending on the frequency for the FEM and the modal model: (a) number of degrees of freedom; (b) number of operations.

The estimation of the number of operations required in order to solve the linear systems of equations is shown in Fig. 6(b). The system matrices for the FEM are supposed to be banded of size $n$ and bandwidth $b$ so the number of operations is approximately bounded by $O\left(n b^{2}\right)$ according to [23] (if $n \gg b$ ). The bandwidth $b$ is estimated as the number of nodes in a two-dimensional cross-section of the problem in the $x-z$ plane.

For the modal model two limit situations are considered. On the one hand, the worst case when the linear system is solved monolithically and a full matrix is used. 
In that situation the number of operations required is $8 n^{3} / 3$ [23] (direct solver with complex arithmetic). The number of operations required by the three-dimensional FEM is larger. The advantages of the modal model are more important for mid and high frequencies.

On the other hand a better situation where the linear system can be solved by block Gauss-Seidel iterations [27]. In this case it is assumed that the major operations in each iteration are 8 matrix-vector products that require $n \times m$ operations (here $n$ is the number of eigenfunctions in the room and $m$ the number of functions used in order to interpolate the pressure field inside the slit) and 4 resolutions of a diagonal system of equations. These operations are multiplied by 10 which is a reasonable number of iterations based on the experience with the use of the modal model. If this block iterative strategy can be applied, the number of operations required in the modal model is reduced (with respect to the monolithic approach). The method can be used for most of the frequencies analysed. As described in [27] it can suffer convergence problems only for some particular frequencies that are very close to the eigenfrequencies or in strongly coupled situations. Realistic values of damping required for modelling overcome the convergence problems around eigenfrequencies. There is not strong coupling for most of the connections analysed here. In any case, for those frequencies where the iterative strategy diverges, the monolithic approach has been used.

The three-dimensional problem used here will be taken as model problem in the remainder with the following parameters unless otherwise stated:

- The dimensions of the sending room are: $L_{x}=2 \mathrm{~m}, L_{y}=3.5 \mathrm{~m}$ and $L_{z}=3 \mathrm{~m}$, and for the receiving room $L_{x}=2.5 \mathrm{~m}, L_{y}=3.5 \mathrm{~m}$ and $L_{z}=3 \mathrm{~m}$.

- For each room walls at $y=0.0 \mathrm{~m}$ and $y=3.5 \mathrm{~m}$ are considered purely reflecting and the other walls with an absorption of approximately $6 \%$ (according to Table 1).

- A point sound source (with a source strength $q_{s}=0.001 \mathrm{~m}^{3} / \mathrm{s}$ ) is placed at position $X_{s}=0.3 \mathrm{~m}, Y_{s}=0.6 \mathrm{~m}, Z_{s}=0.4 \mathrm{~m}$ in the sending room.

- A slit with default dimensions $3.5 \mathrm{~m}$ in length, $0.01 \mathrm{~m}$ in width and $0.05 \mathrm{~m}$ in depth is considered. It is placed at the bottom of the separating surface $\left(Y_{0}=0\right.$ and $Z_{0}=h / 2$, according to the notation used in Fig. 1). The slit width is specified in every case.

- The Hz frequency interval between consecutive calculations is: 1 (for frequencies below $562 \mathrm{~Hz}$ ), 2 (for frequencies between $562 \mathrm{~Hz}$ and $1778 \mathrm{~Hz}$ ), 5 (for frequencies between $1778 \mathrm{~Hz}$ and $2239 \mathrm{~Hz}$ ) and 10 (for frequencies between $2239 \mathrm{~Hz}$ and $3548 \mathrm{~Hz})$.

\subsection{Comparison with Gomperts' model and laboratory mea- surements}

A group of laboratory measurements of the sound reduction index of slits is reported in [11]. Twenty slits with variable depth $L_{\xi}$ and width $h$ were considered. The same 
twenty slits are analysed here with the goal of placing the proposed modal model in a well known context where experimental results and a simplified model are available. The exact dimensions of the laboratory rooms are not mentioned in [11]. Its volume is $100 \mathrm{~m}^{3}$ for the sending room and $85 \mathrm{~m}^{3}$ for the receiving. In order to have these volumes, the dimensions of the sending room are: $L_{x}=5.71 \mathrm{~m}, L_{y}=5 \mathrm{~m}$ and $L_{z}=3.5$ $\mathrm{m}$, and for the receiving room $L_{x}=4.86 \mathrm{~m}, L_{y}=5 \mathrm{~m}$ and $L_{z}=3.5 \mathrm{~m}$. According to the experimental set up, the tested wall with slit was placed in a square window between two rooms in the laboratory. The slit was in the middle of that window. Taking into account also the values of parameters $m$ and $n$ in the formulation we decide to place the slit in the middle of the wall with a length $L_{\eta}=1.94 \mathrm{~m}$ (the same slit length used in the laboratory experiment), $Y_{0}=\left(L_{y}-L_{\eta}\right) / 2$ and $Z_{0}=L_{z} / 2$, according to the notation used in Fig. 1.

The sound reduction index is calculated from the model proposed here as

$$
R=L_{S}-L_{R}+10 \log _{10}\left(\frac{h L_{\eta}}{A_{R}}\right)
$$

with $A_{R}=\sum_{i} S_{i} \alpha_{i}$ the absorption in the receiving room, where $S_{i}$ is the surface of each absorbing (Robin) wall and $\alpha_{i}$ its absorption coefficient (according to Table 1 ). This formula is adapted from the version used for the sound reduction index of a wall between rooms. The slit surface is considered in order to calculate the incident acoustic energy instead of the wall surface.

The results are presented in Figs. 7, 8, 9 and 10 corresponding to slit depths $L_{\xi}=1.5 \mathrm{~mm}, 20 \mathrm{~mm}, 50 \mathrm{~mm}$ and $100 \mathrm{~mm}$ respectively. In each figure three curves are shown: i) laboratory measurements of [11]; ii) predictions by means of the Gomperts' model presented in [10] reproducing the same cases reported in Figs. 2, 3, 4 and 5 of [11]; iii) the results obtained in this work. 

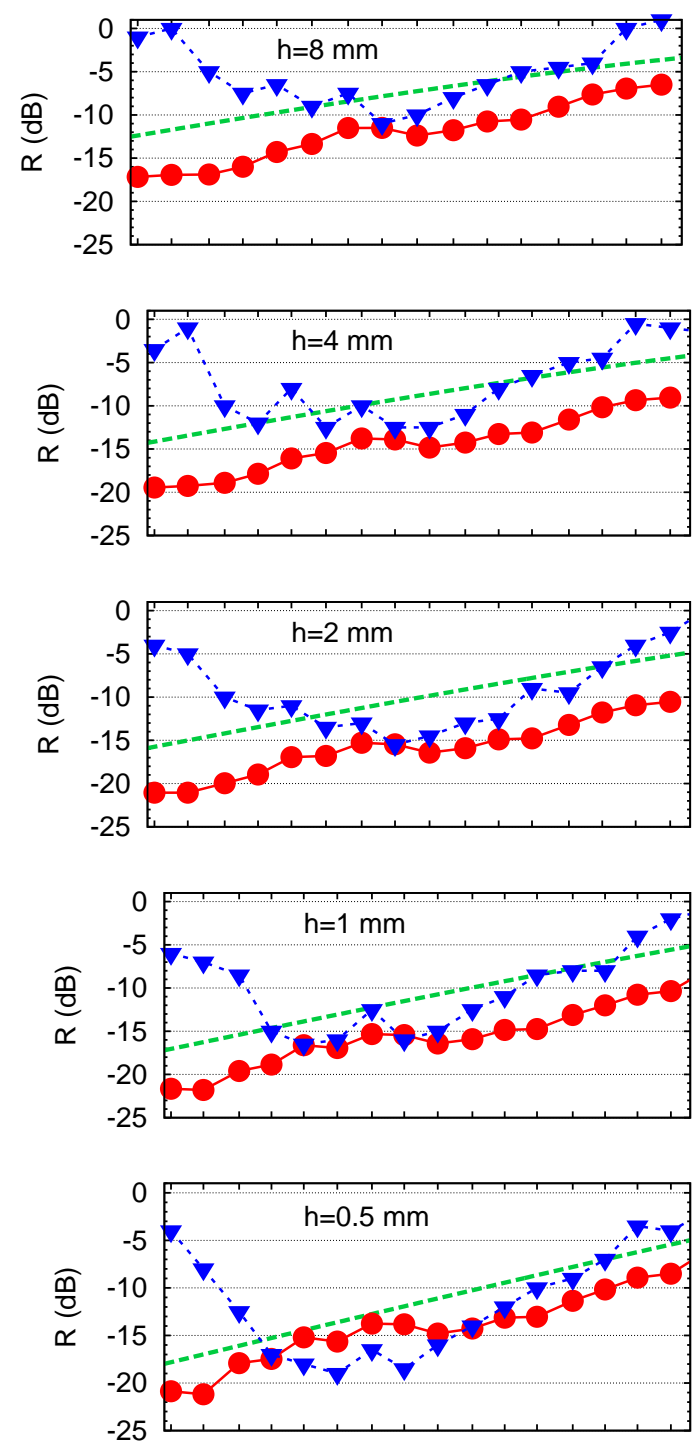

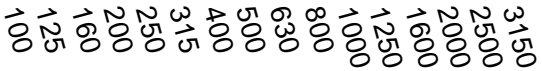

$\mathrm{f}(\mathrm{Hz})$

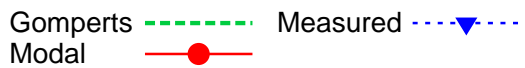

Figure 7: Comparison of the modal analysis results, the Gomperts' model predictions and the laboratory measurements reported in [11]: $L_{\xi}=1.5 \mathrm{~mm}$.
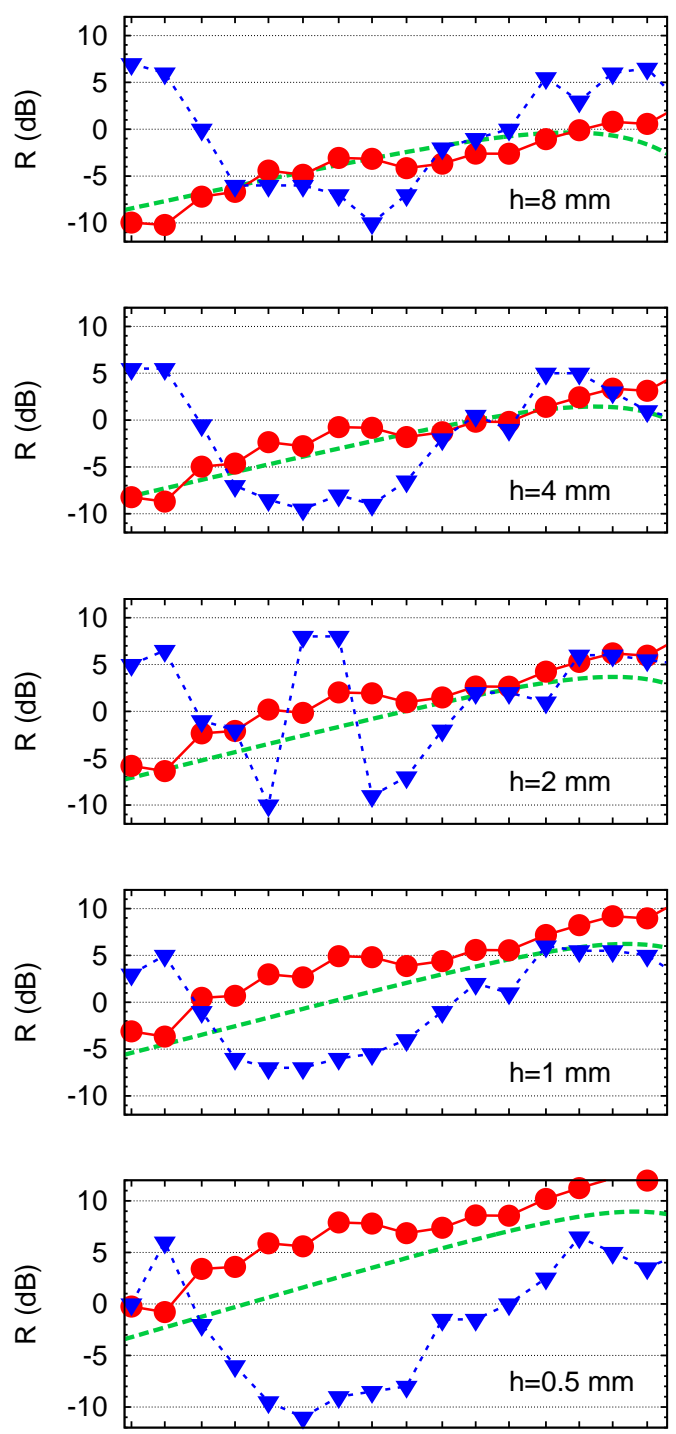

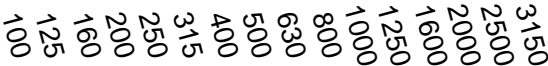

$\mathrm{f}(\mathrm{Hz})$

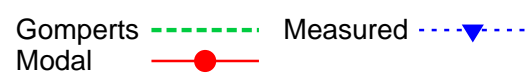

Figure 8: Comparison of the modal analysis results, the Gomperts' model predictions and the laboratory measurements reported in [11]: $L_{\xi}=20 \mathrm{~mm}$. 

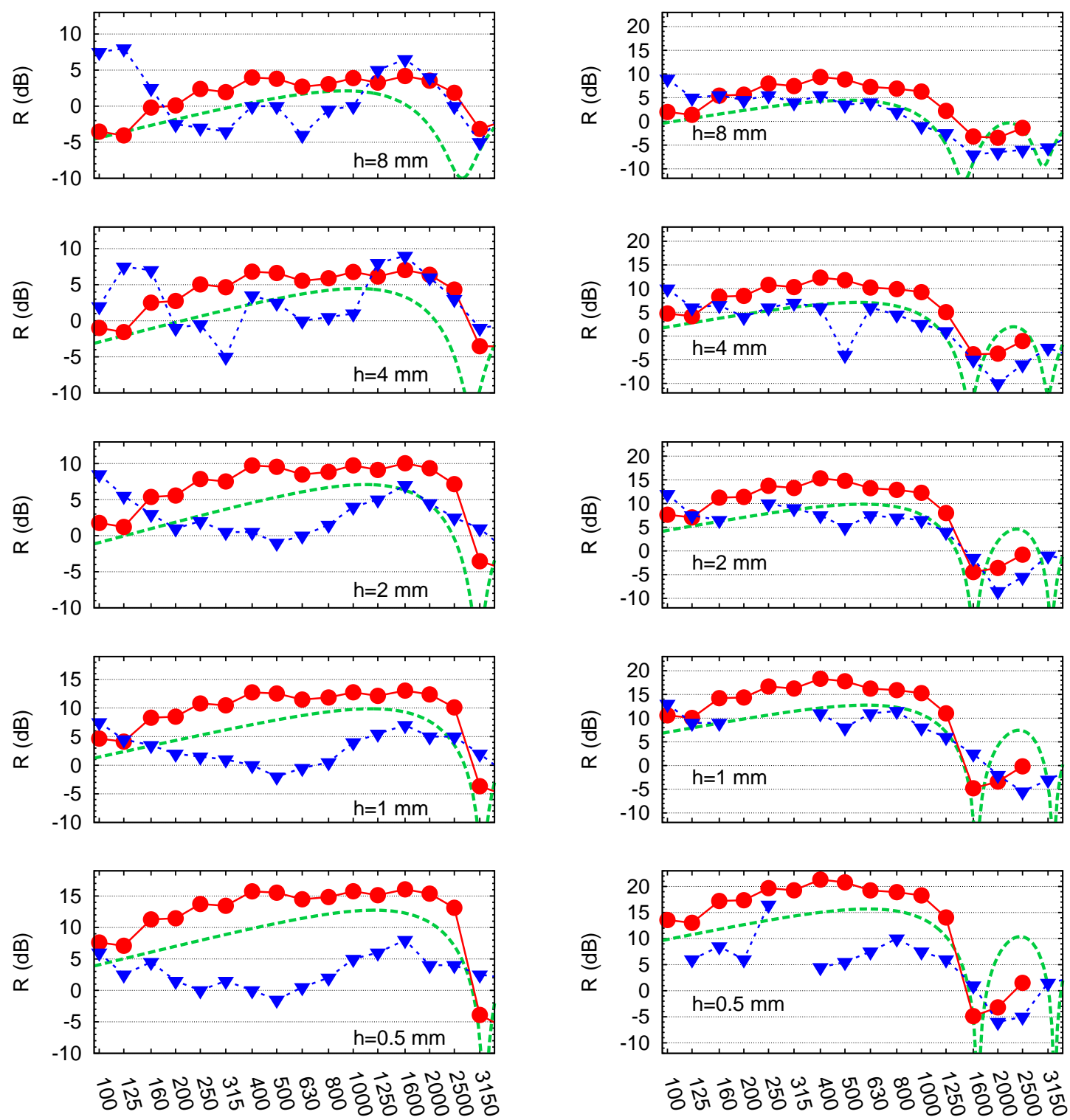

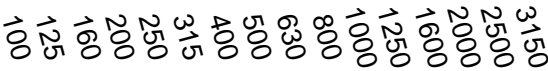

$f(\mathrm{~Hz})$

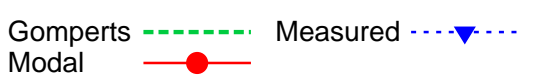

$f(\mathrm{~Hz})$

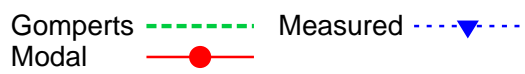

Figure 9: Comparison of the modal anal- Figure 10: Comparison of the modal analysis results, the Gomperts' model predictions and the laboratory measurements reported in [11]: $L_{\xi}=50 \mathrm{~mm}$. ysis results, the Gomperts' model predictions and the laboratory measurements reported in [11]: $L_{\xi}=100 \mathrm{~mm}$. 
Some of the details of the experiment (room dimensions and values of absorption or reverberation time) are not reported in [11]. This always makes more difficult the task of reproducing the experiment with numerical models. However, the volume of the laboratory rooms was quite large $\left(100 \mathrm{~m}^{3}\right.$ for the sending room and $85 \mathrm{~m}^{3}$ for the receiving). For this reason, the pressure fields should be diffuse even at mid frequencies and the dependence of the results on the particular room modes low. In any case, the obtained agreement in the general trend of the results and the order of magnitude is satisfactory.

It can be said that the modal model results follow the general tendency of Gomperts' model. The largest differences are found for the very thin $1.5 \mathrm{~mm}$ slit (see Fig. 7). Also after the first slit resonance predicted by Gomperts' model (see Fig. 10 in the case with $L_{\xi}=100 \mathrm{~mm}$ ), the behaviour is quite different. The modal model results do not suffer these strong dips caused by resonances. In that sense, the modal model is closer to the laboratory measurements.

An important difference between Gomperts' model and the proposed modal model is that in the first case the slit is placed between unbounded acoustic domains. In consequence the sound reduction index curve depends only on the slit resonances (room resonances are not considered). In order to calculate these resonances, the end correction factor of the aperture must be known. In the modal model, the sound reduction index depends on the resonances of all the subsystems (rooms and slit). The results in that second case must be averaged in order to obtain some clear information. In the experimental measurements reported in [11], the first slit resonance seems to be important but the effect of the subsequent resonances seems to be overestimated by Gomperts' model. The modal model results are in better agreement with experimental measurements in this frequency range where other factors different from the slit resonances are considered.

In the low-frequency range, the sound reduction index curve proposed by Gomperts' model decreases monotonically with decreasing frequency. On the contrary, the modal model prediction often tends to residual values that oscillate depending on the modal behaviour of the system. The agreement with experiments in that zone highly depends on the properties (dimensions, shape and absorption distribution) of the rooms.

The modal model and the Gomperts' model have also been compared for the case where the slit is placed in an edge of the room. The rooms defined in Section 3.1 are considered now and the slit is placed in the bottom edge. In the results shown in Fig. 11 it can be seen that both models follow the same trend. The main general difference is that after the first slit resonance the ordering of the curves is different (see Fig. 11(d) for frequencies over $1600 \mathrm{~Hz}$ ). In fact, the behaviour is different as explained for the cases of the slit in the middle of the wall. Differences with Gompert's model are larger for thinner slits.

Other aspects (slits of different shape, different positions of the slit in the wall, slits of shorter length with respect to wall size,...) can also be considered by the modal model without requiring a modification on the formulation. This is not the case of Gomperts or other similar models. The versatility of the modal model presented here is one of its main advantages. 


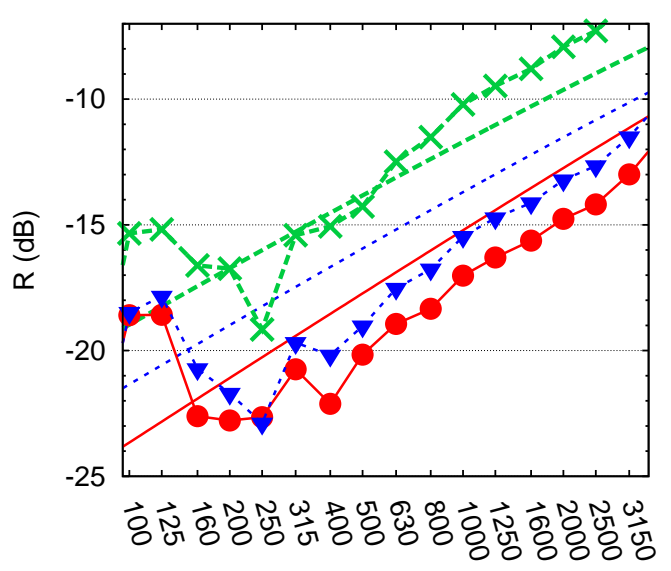

$\mathrm{f}(\mathrm{Hz})$

(a)

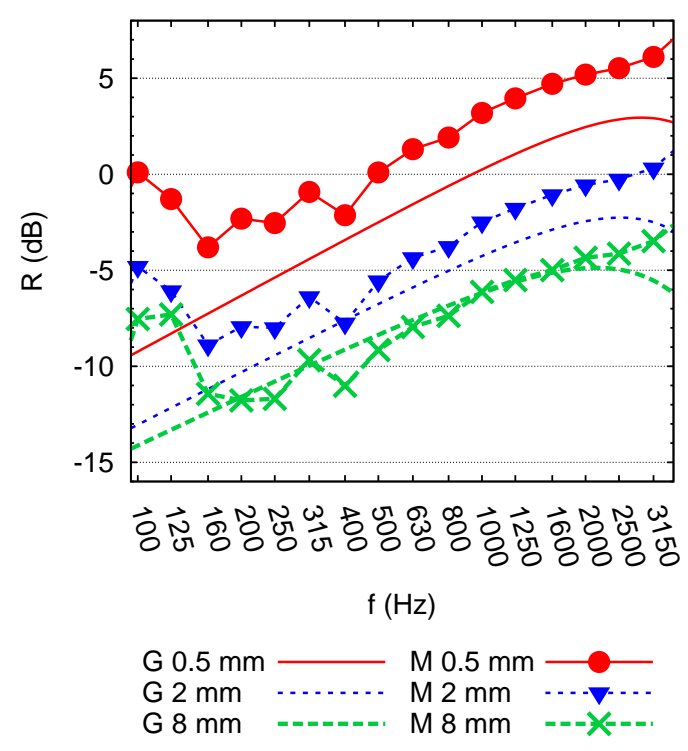

(c)

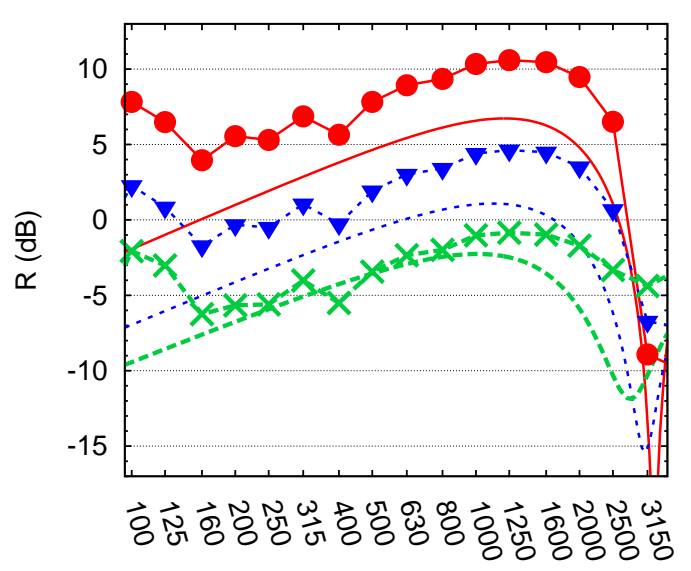

$\mathrm{f}(\mathrm{Hz})$

(b)

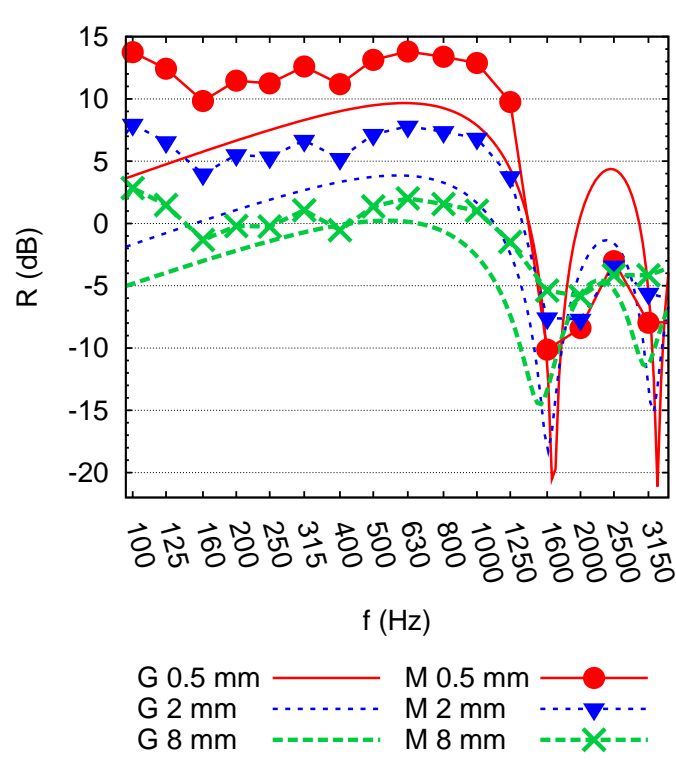

(d)

Figure 11: Comparison of the modal analysis results (M) and the Gomperts' model $(\mathrm{G})$ when the slit is placed in the bottom of the room for several values of slit width ( $\alpha=10 \%$ ). Four different values of slit depth: (a) $L_{\xi}=1.5 \mathrm{~mm}$; (b) $L_{\xi}=50 \mathrm{~mm}$; (c) $L_{\xi}=20 \mathrm{~mm}$; (d) $L_{\xi}=100 \mathrm{~mm}$.

\subsection{Influence of slit length}

The influence of slit length is analysed now. The same model problem defined in Section 3.1 is considered.

Most of the models found in the literature for straight slits assume that the slit is infinite and thus, the problem can be reduced to the analysis of a two-dimensional cross-section. By combining this simplification with other hypotheses, an analytical 
approach leads to simplified expressions of the sound reduction index. In most of the models these expressions are simplified but still need the use of a computer to be evaluated (for example, very often a large number of evaluations depending on the incident pressure angle are required). Also in [11] several slit lengths (shorter than $2.0 \mathrm{~m}$ ) are tested in order to justify that when the acoustic wave length is shorter than the slit length, the dependence of the sound reduction index on this length can be neglected.

A parametric analysis in order to verify the influence of the slit length $L_{\eta}$ on the sound reduction index predictions is done. The results are shown in Fig. 12. The values of the slit width $h$ are representative of slits under doors $(h=1 \mathrm{~mm})$ or small openings needed in order to pass wires, pipes, ventilation systems, etc $(h=50 \mathrm{~mm})$.

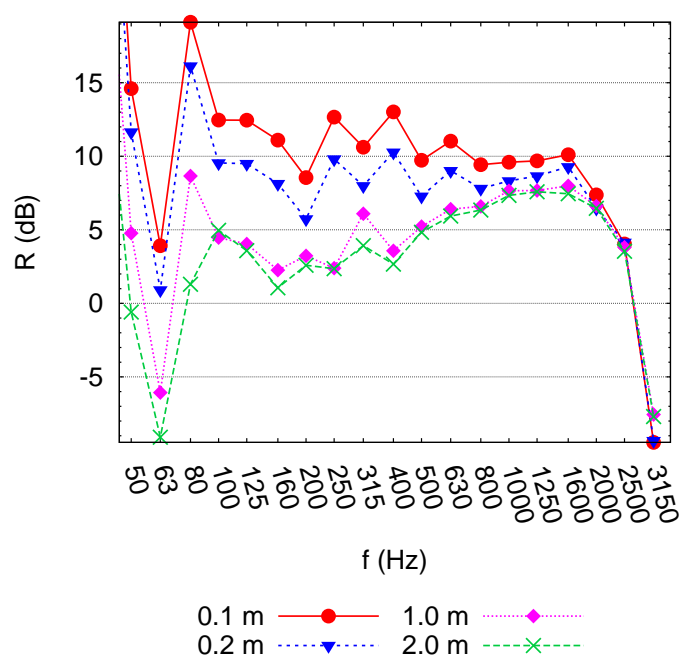

(a)

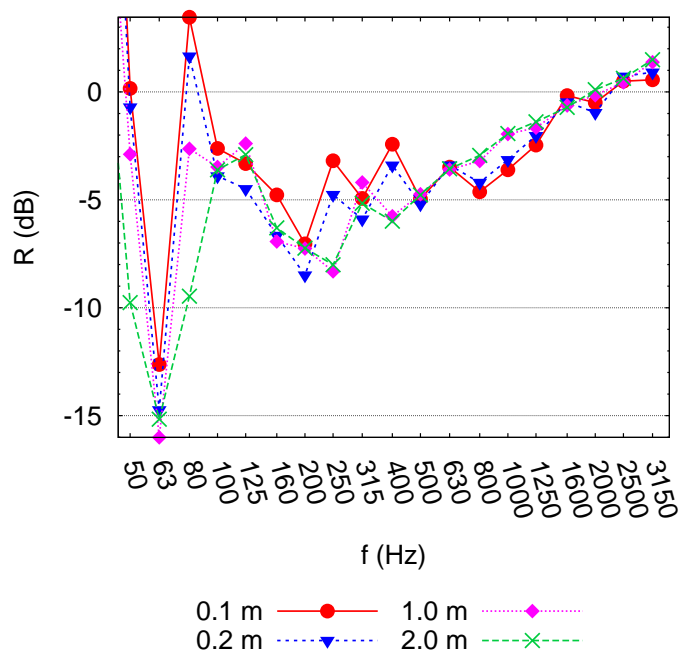

(b)

Figure 12: Influence of the slit length $L_{\eta} . \quad\left(\alpha=10 \%, L_{\xi}=0.05 \mathrm{~m}\right)$ Two different values of slit width: (a) $h=1 \mathrm{~mm}$; (b) $h=50 \mathrm{~mm}$.

It can be concluded that the influence of the slit length highly depends on the slit width. The thinner the slit, the larger the dependence on the slit length. This is in agreement with the results shown in [28]. The dependence on $L_{\eta}$ is important for the lower frequencies. This frequency range is larger for shorter slits.

\subsection{Influence of room properties: size and absorption}

Due to the nature of the model a possible influence of the room properties on the slit sound reduction index can be studied. Two different aspects are considered: the room size and the acoustic absorption. These parameters are taken into account by means of Eq. (47).

The room dimensions described at the end of Section 3.1 are taken as reference. The dimensions of the sending and receiving rooms have been modified in order to study the influence of room size in the sound reduction index. The analysis goes in 
the line proposed in [19] for the sound transmission of a rigid wall at low frequencies. $L_{y}=3.5 \mathrm{~m}$ is constant in all situations. Then, with constant dimensions of the sending room, twenty different receiving rooms are considered $\left(L_{x}=2,2.5,3,4\right.$ and $5 \mathrm{~m}, L_{z}=2,3,4$ and $5 \mathrm{~m}$,). The same is done with constant dimensions of the receiving room and the same twenty combinations of $L_{x}$ and $L_{z}$ are considered in the sending room. The analysis has been repeated for each value of acoustic absorption in Table 1 in all the walls of the room. The sound source is always placed at the position $\left(0.15 L_{x} ; 0.17 L_{y} ; 0.13 L_{z}\right)$. The slit is placed at the bottom of the wall.

Fig. 13 shows the effect of room dimensions on the sound reduction index. Several outputs are plotted: i) Averaged sound reduction index $\left(R_{\text {var. sending }}\right.$ is the sound reduction index with variations in the size of the sending room while $R_{\text {var. receiving }}$ is obtained with variations in the size of the receiving room); ii) standard deviation $\sigma$ of all calculated values of $R$; iii) maximum and minimum values of sound reduction index for each frequency. Again, the results are compared with Gomperts' model. The agreement with Gomperts' model is quite similar in both cases.

The standard deviation is around $3-5 \mathrm{~dB}$ at low frequencies but decreases fast and at mid frequencies is not larger than $2 \mathrm{~dB}$. Variations in the size of the receiving room have less influence in the variation of sound reduction index. This can be explained because the calculation of $R$ takes into account the size and absorption of the receiving room. Nevertheless, the formula assumes a diffuse field in the sending room which is not the case of the smallest rooms considered at low-frequencies. The deviations of $R$ (maximum and minimum values that are shown with bars) can be larger than 10 $\mathrm{dB}$ at low frequencies for certain combinations of room dimensions. This is in close relationship with the distribution of modes in each one-third octave frequency band. At mid frequencies this variation is still quite important $( \pm 2 \mathrm{~dB})$.
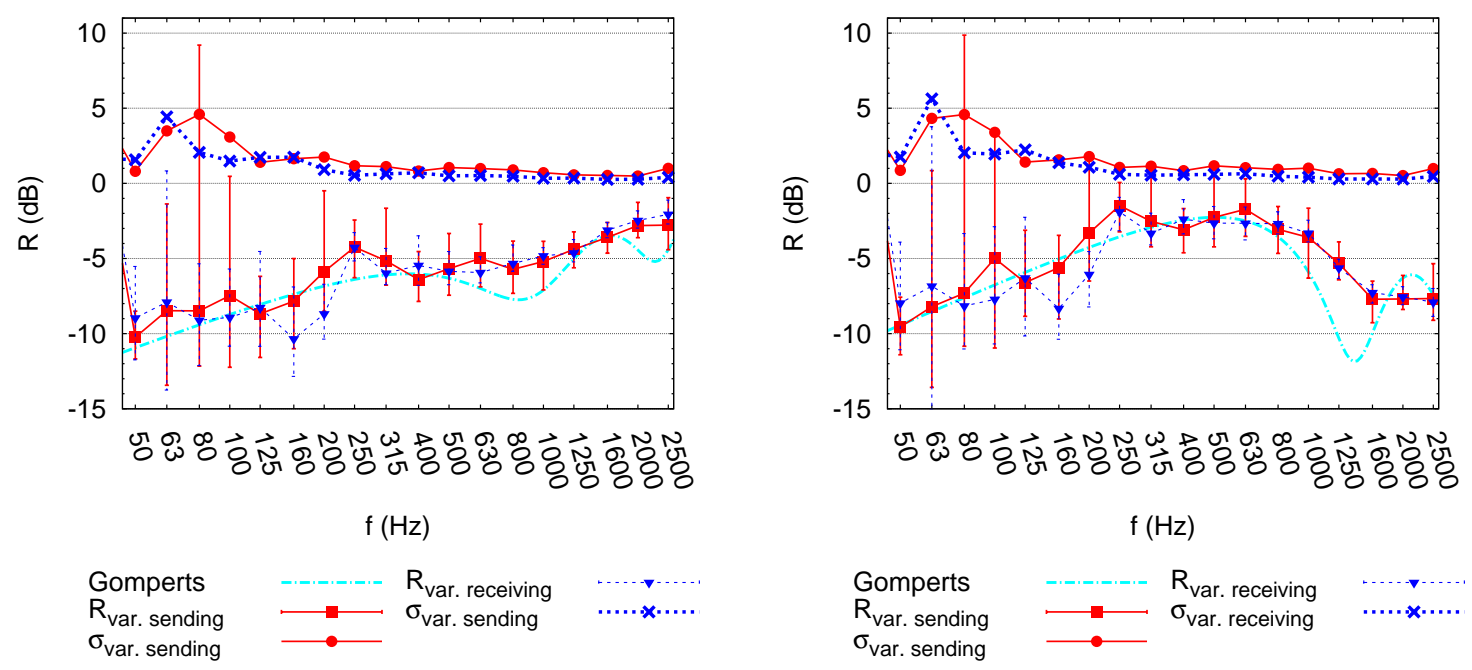

Figure 13: Influence of the room size in the calculation of the sound reduction index $(R)$ of several slit widths: (a) $h=0.05 \mathrm{~m}$; (b) $h=10 \mathrm{~mm} .\left(\alpha=10 \%, L_{\xi}=0.1 \mathrm{~m}\right)$.

Fig. 14 shows the influence of the room absorption in the sound reduction index. Eq. (47) takes into account the absorption of the receiving room in order to transform 
the sound level difference, which is a very absorption dependent output, to sound reduction index, which is less dependent on the absorption. However, differences can be of $2 \mathrm{~dB}$ for the case of $30 \%$ of absorption. An hypothesis assumed in order to derive Eq. (47) is the existence of a diffuse pressure field, which is sometimes not fully satisfied in the analysed situations. Another possible cause of these small differences between the sound reduction index curves is the fact that the eigenfunctions chosen here become less adequate if the absorption is increased.

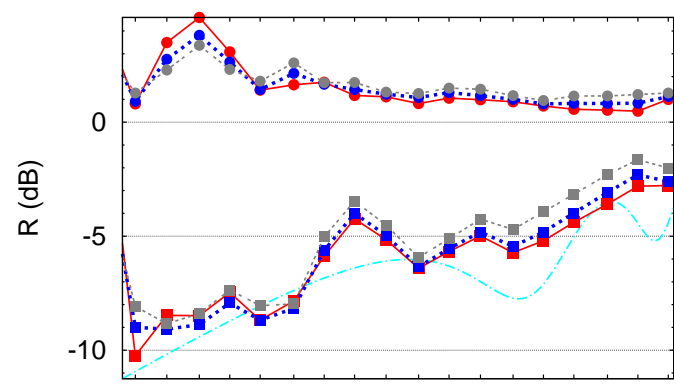

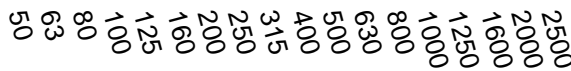

$f(\mathrm{~Hz})$

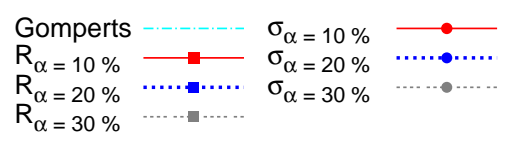

(a)

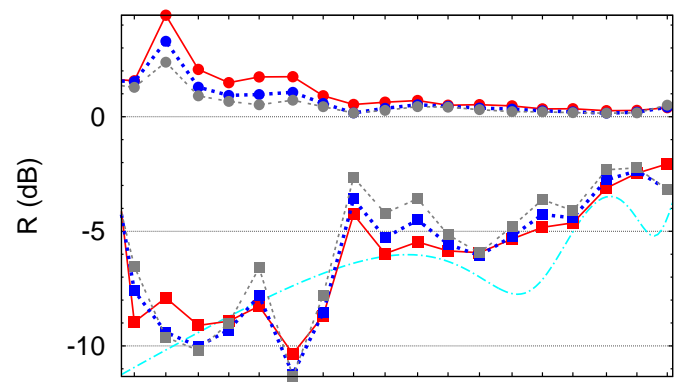

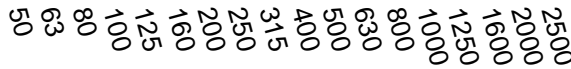

$f(\mathrm{~Hz})$

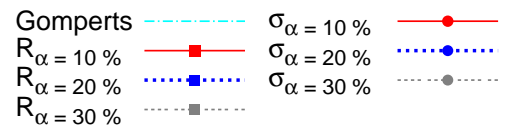

(c)

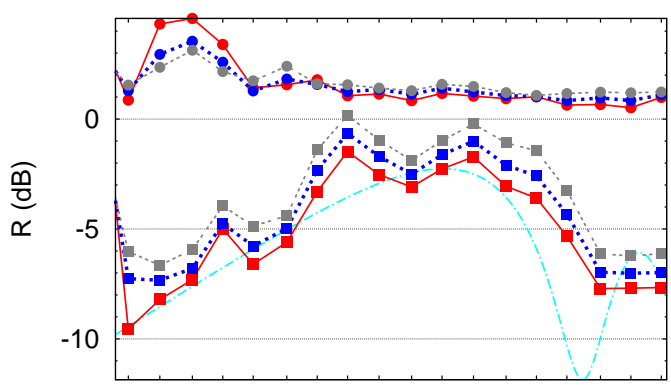

গু

$f(\mathrm{~Hz})$

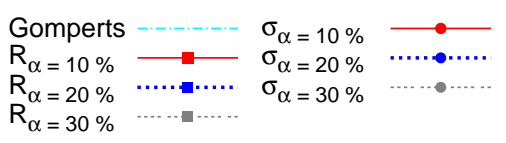

(b)

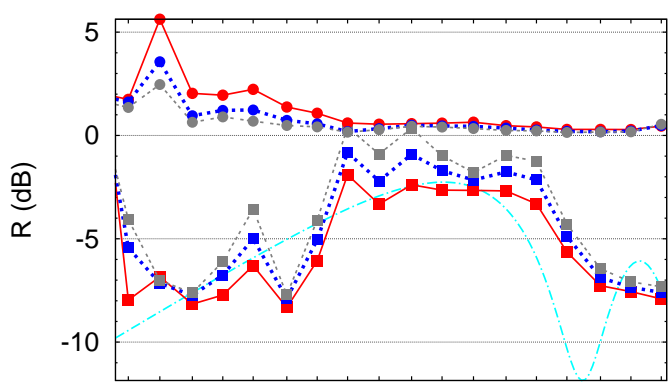

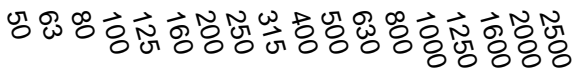

$f(\mathrm{~Hz})$

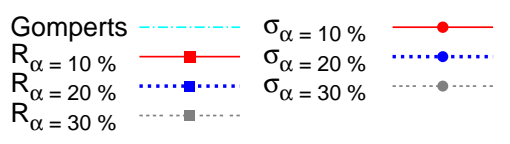

(d)

Figure 14: Sound reduction index $R$ calculated for several values of room absorption $\left(L_{\xi}=0.1 \mathrm{~m}\right.$ ): (a) $h=0.05 \mathrm{~m}$ and variation of sending room size; (b) $h=0.01 \mathrm{~m}$ and variation of sending room size; (c) $h=0.05 \mathrm{~m}$ and variation of receiving room size; (d) $h=0.01 \mathrm{~m}$ and variation of receiving room size. 


\subsection{Influence of opening position and combination of two holes}

One of the advantages of the model considered here with respect to the solutions for openings and slits available in the literature is that the influence of the location of the opening can be analysed. Four different situations are considered: i) hole in the middle of the wall; ii) hole in the corner of the wall; iii) hole in an edge of the room; iv) hole in an intermediate position between middle and corner (quarter). The details on the position can be found in Table 2 and Fig. 15. The hole/opening is treated as a three-dimensional connecting element (see the top connection in Fig. 1).

\begin{tabular}{ccc} 
Position & $Y_{0}$ & $Z_{0}$ \\
\hline Middle & $\left(L_{y}-L_{\eta}\right) / 2$ & $\left(L_{y}-L_{\mu}\right) / 2$ \\
Corner & $0.1 \mathrm{~m}$ & $0.1 \mathrm{~m}$ \\
Edge & 0 & $\left(L_{z}-L_{\mu}\right) / 2$ \\
Quarter & $\frac{L_{y}}{4}-\frac{L_{\eta}}{2}$ & $\frac{L_{z}}{4}-\frac{L_{\mu}}{2}$
\end{tabular}

Table 2: Position of the opening in the wall.

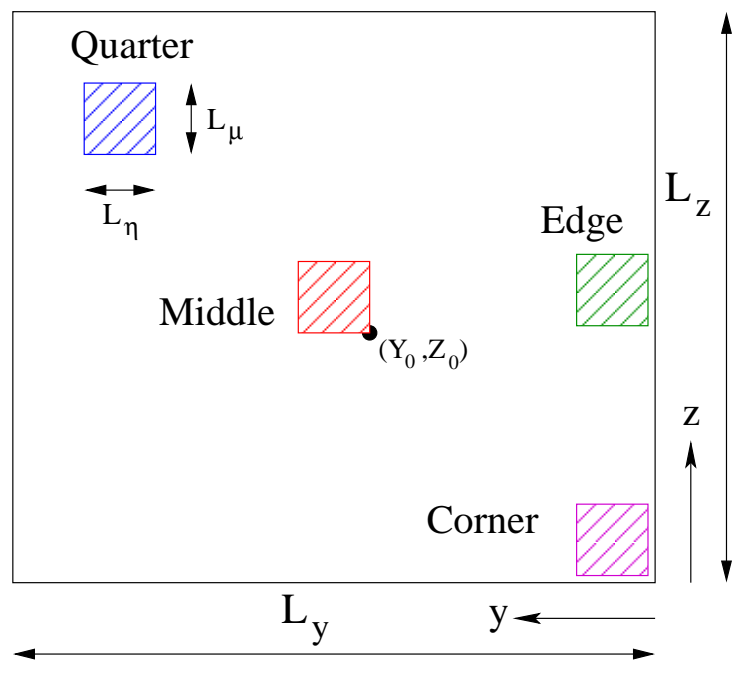

Figure 15: Sketch of the wall with the four positions of the opening.

The sound reduction index for the four configurations is plotted in Fig. 16. The sound reduction index predictions have been compared with the formula for normal incident waves and circular holes proposed by [35] (see also [28] or [14]). For small holes the difference between a circular and square shapes can be neglected as studied in [25]. Wilson and Soroka's model represents an upper bound for the results obtained with the modal model. There is also coincidence in the first sound reduction index dips caused by hole resonances. However, they are more pronounced and 'ideal' in Wilson and Soroka's model than in the modal model and for high frequencies the modal model tends to a more or less flat curve without peaks. 
The position of the hole in the wall highly influences the sound reduction index value. The worst case is always with the hole in the corner followed by holes in the edge. The sound transmission performance is rather affected by the spatial distribution of the exciting pressure field acting on the hole surface (sending side). There is no nodal plane or line going through the corner. The hole is therefore excited by a maximum of oblique incidence waves which decrease the sound reduction index (compared to normal incidence pressure waves which lead to higher values of insulation). Acoustic modes having a nodal plane or a nodal line in the middle of the wall do not contribute to the excitation field. For this reason, the highest insulation is always found for holes in the middle of the wall. This is more relevant at low and mid frequencies (more or less below $900-1500 \mathrm{~Hz}$ in this example but specially for those frequencies lower than the first slit resonance). For high frequencies, all cases tend to provide similar values of sound reduction index.

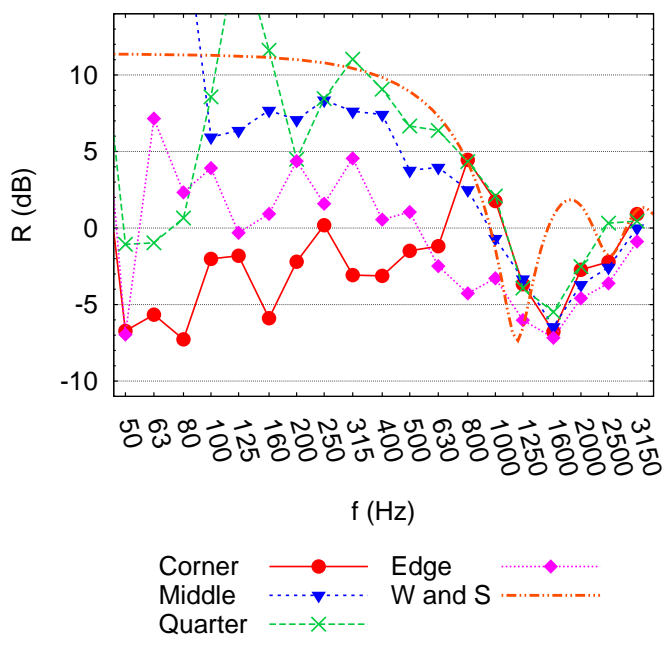

(a)

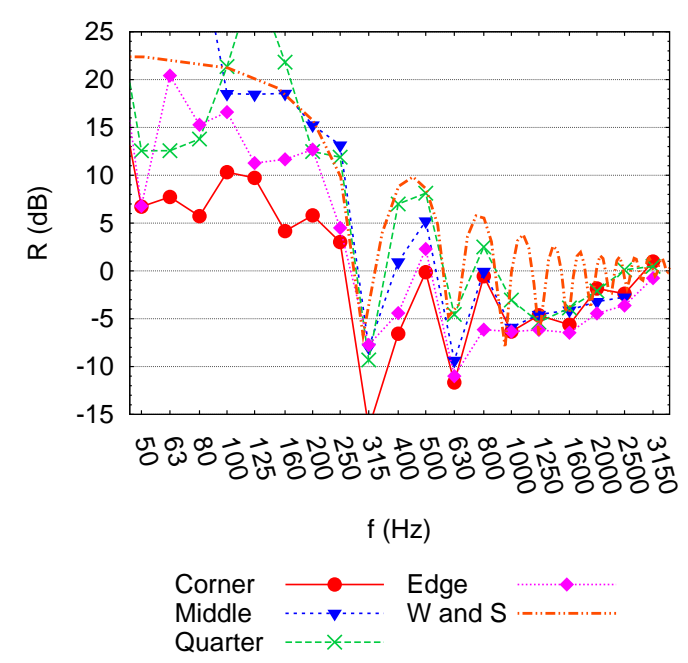

(b)

Figure 16: Influence of the opening position. Square opening of side $L_{\eta}=0.05 \mathrm{~m}$ ( $\alpha=10 \%$ ), comparison with [35] model (W and S): (a) $L_{\xi}=0.1 \mathrm{~m}$ (b) $L_{\xi}=0.5 \mathrm{~m}$.

The interaction between two holes has also been studied. The modal model prediction including two holes at the same time has been compared with a combination of the sound reduction indices of single holes. This addition can be done for the case of $n$ holes as

$$
R_{\text {total }}=-10 \log _{10}\left(\sum_{j}^{n} \frac{S_{j} 10^{-\frac{R_{j}}{10}}}{S_{\text {total }}}\right)
$$

where $R_{j}$ is the sound reduction index of a single hole of surface $\mathrm{S}_{j}$ and $\mathrm{S}_{\text {total }}$ is the surface of all the holes.

This is illustrated by means of two examples: i) holes in Middle and Corner positions; ii) holes in the edge of the room. In the first example, the holes are in very different positions and, as shown in Fig. 16, this can lead to different sound reduction index curves. In the second case, the position of the holes is chosen in order to avoid a possible masking of the coupling effect. 
Fig. 17 shows the study of two situations. In the first one, holes in Corner and Middle positions are considered. Due to the room dimensions, we can ensure that the separation between them is larger than an acoustic wavelength at low frequencies. In the second one, the Corner hole is replaced by a hole which is very close to the Middle hole. The separation between them is $7.5 \mathrm{~cm}$ in horizontal and vertical directions, which is smaller than an acoustic wavelength in a wide frequency range. The sound reduction index curves in Fig. 17 (a) correspond to the following hole configurations:

- Isolated holes at positions: Corner (C), Middle (M) and displaced $7.5 \mathrm{~cm}$ from the Middle $\left(\mathrm{M}^{*}\right)$

- Two holes at the same time: Corner and Middle positions (Calc.: C+M) and both in the centre of the wall (Calc.: $\mathrm{M}+\mathrm{M}^{*}$ )

- Prediction of the insulation of two holes by means of the single hole results and Eq. (48): Corner and Middle positions (Combined: $\mathrm{C}+\mathrm{M}$ ) and both in the centre of the wall (Combined: $\mathrm{M}+\mathrm{M}^{*}$ )

When the separation between holes is large $(\mathrm{C}+\mathrm{M})$, the direct calculation or combination of results for isolated holes provide very similar results (except at very low frequencies where some particular mode can be excited). The combined curve is always between the curves corresponding to the isolated holes. On the contrary, when the holes are very close $\left(\mathrm{M}+\mathrm{M}^{*}\right)$, differences between both 'calculated' and 'combined' results can be important. To avoid a prediction method considering both holes at the same time leads to an overestimation of the sound reduction index.

Small square openings of side $L_{\eta}=0.01 \mathrm{~m}$ in Edge position are considered in the second example. In addition to the Edge hole described in Table 2, three other holes where $Z_{0}$ is increased 5,10 and $50 \mathrm{~cm}$ are taken into account. Fig. 17(a) shows the insulation of these holes when they are alone. Only for the last one $(50 \mathrm{~cm})$ the sound reduction index curve is quite different. This is due to the different position of the hole in the wall. However, for the other three cases (centred in the edge, $5 \mathrm{~cm}$ and $10 \mathrm{~cm}$ ), the three sound reduction index curves are very similar and we can ensure that future differences, if any, will not be caused by the position of the hole in the room but by the possible interaction. Fig. 17(b) shows the difference between sound reduction index of two holes evaluated as combination of the sound reduction index of single isolated holes and the sound reduction index calculated in a problem with two holes at the same time.

For the examples studied here, the coupling effect between holes is relevant while the separation between holes is around $0.25-0.5$ times the acoustic wavelength. Neglecting the coupling effect for this cases of close holes can lead to overestimations of the sound reduction index around $3 \mathrm{~dB}$. The effect of hole interaction can be even more relevant in other problems like the sound transmission through micro perforated plates [7].

Fig. 19 shows the effect of the hole area in the sound reduction index. $R$ is larger for openings with smaller area (the same trend as for the slit length). This difference is more important for less transmissive situations: holes in the middle of the wall and frequencies not coincident with resonances of the opening. 


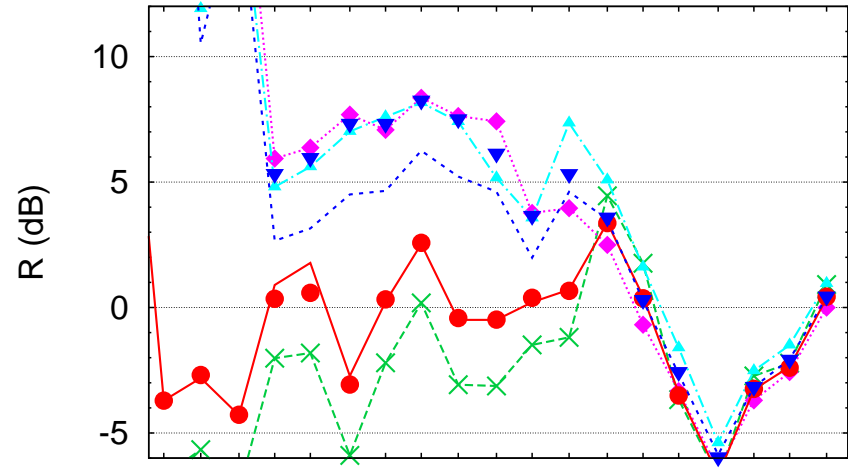

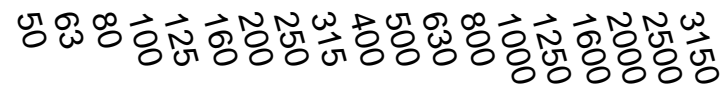

$\mathrm{f}(\mathrm{Hz})$

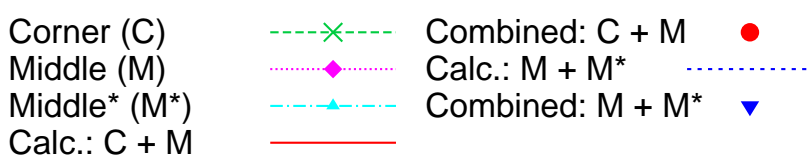

(a)

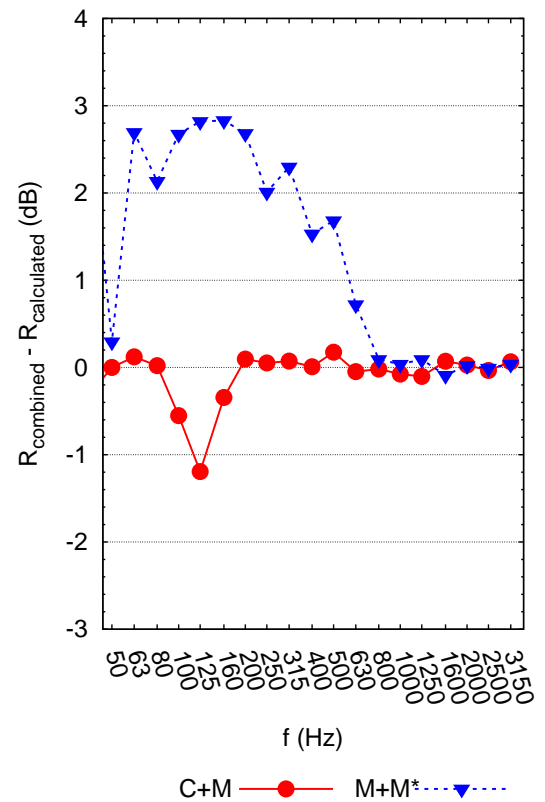

(b)

Figure 17: Sound transmission through two holes, compared with the combination of results for single holes. Square opening of side $L_{\eta}=0.05 \mathrm{~m}\left(\alpha=10 \%, L_{\xi}=0.1 \mathrm{~m}\right)$ : (a) Sound reduction index for single holes (in Middle (M), Corner (C) and almost Middle $\left(\mathrm{M}^{*}\right)$ positions) and two holes (calculated in a model including two holes at the same time or combined with results of single holes); (b) Difference between 'calculated' and 'combined' sound reduction index.

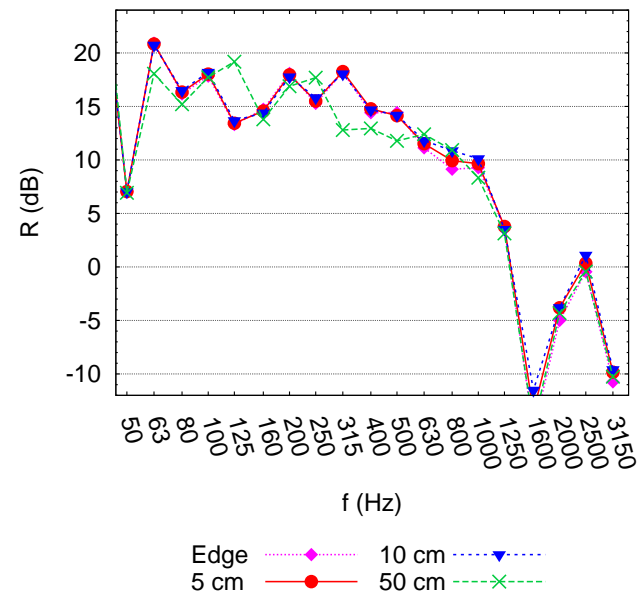

(a)

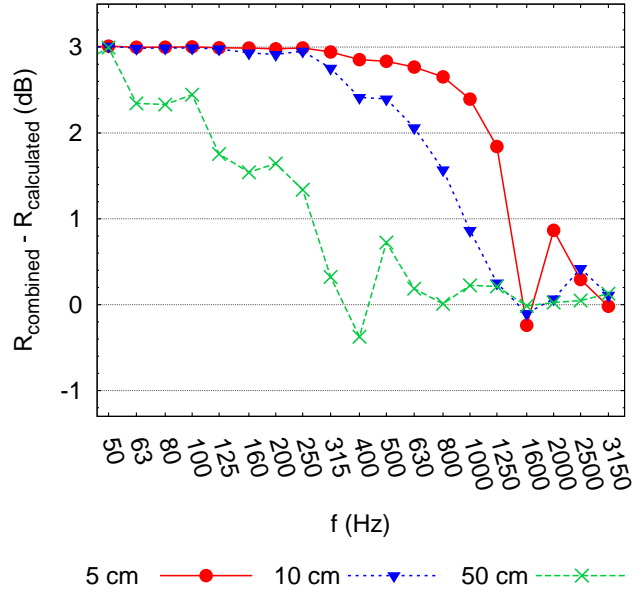

(b)

Figure 18: Square opening of side $L_{\eta}=0.01 \mathrm{~m}\left(\alpha=10 \%, L_{\xi}=0.1 \mathrm{~m}\right)$ : (a) Sound reduction index of a single hole in the Edge position or also in the edge but displaced 5, 10 and $50 \mathrm{~cm}$ from the centre; (b) Difference between 'calculated' and 'combined' sound reduction index. 


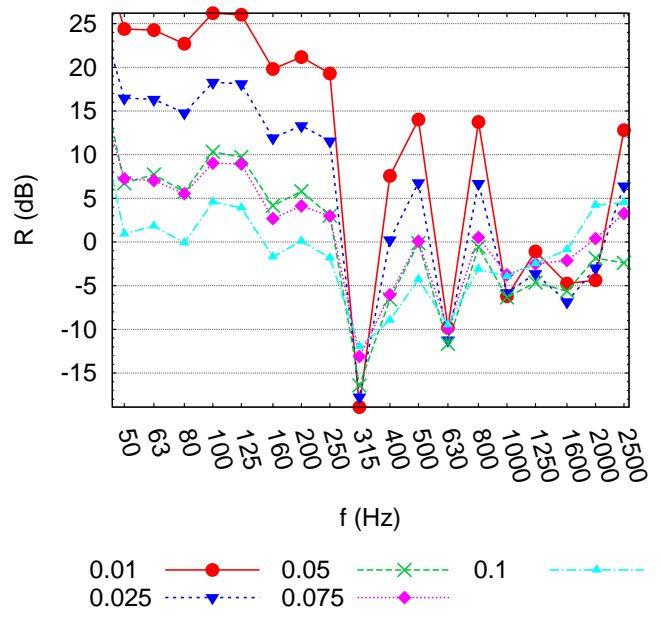

(a)

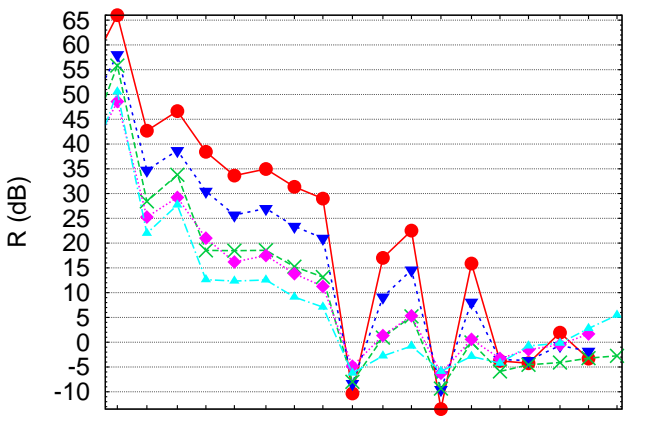

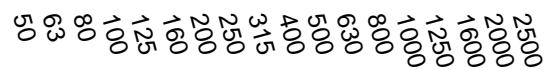

$f(\mathrm{~Hz})$

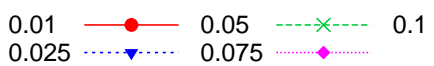

(b)

Figure 19: Influence of the hole area in the sound reduction index for five values of side of the square opening in meters $\left(\alpha=10 \%, L_{\xi}=0.5 \mathrm{~m}\right)$. Two positions of the hole: (a) Corner; (b) Middle. 


\section{Conclusions}

Some conclusions can be obtained from the work presented here:

1. The proposed model can be used in order to predict the sound transmission through slits and openings between cuboid-shaped rooms. It lies between a model that assumes major simplifications that lead to an analytical expression for the sound reduction index of slits and a FEM model of the slit and surroundings. On the one hand, a geometrical description of the problem can be done in detail. On the other hand the formulation has been adapted by using eigenfunctions in order to reduce computational costs when compared to numerical techniques based on element discretisation. As an example and according to the estimations presented in Section 3.1, the number of degrees of freedom required at $1000 \mathrm{~Hz}$ for a three-dimensional FEM model are divided by 50 and the number of operations by a factor between $10^{2}$ and $10^{6}$. This improvement increases for higher frequencies.

2. The slit length is an important parameter that can modify the sound reduction index of slits. This is more important for thin slits.

3. The sound transmission of a hole or an opening depends on the position in the wall. The worst situation (lowest sound reduction index) is found with the hole placed in the corner while the best one is when the hole is in the central part of the wall.

4. Situations where the sound is transmitted through two holes have been considered. It has been seen that the interaction between both holes is very low if the separation between them is large. In that cases, the total sound reduction index can be calculated by combination of the sound reduction indices of the two isolated holes. The interaction between holes is relevant while the separation between them is around $0.25-0.5$ times the acoustic wavelength. Neglecting the coupling effect in that cases of close holes can lead to overestimations of the sound reduction index around $3 \mathrm{~dB}$.

5. The analysis of the sound transmitted between rooms of variable size reveals that room size can be an important aspect. Large variations are found in the low-frequency range. However, they are also important (around $\pm 3 \mathrm{~dB}$ ) in the mid frequency range. This variation of sound reduction index depends more on the variation of the sending room.

6. In the mid and high frequencies, the modal predictions are less sensitive to slit resonances than Gomperts' model. The low-frequency response predicted by the modal model oscillates according to the modes of the system. The sound reduction index values in this frequency range do not follow, in general, a monotonically decreasing trend with decreasing frequency. 


\section{References}

[1] L.H. Bell and D.H. Bell. Industrial noise control. Dekker, New York, 1993.

[2] L.L. Beranek and I.L. Vér. Noise and vibration control engineering. John Wiley and sons, 1992.

[3] T. Bravo and S.J. Elliott. Variability of low frequency sound transmission measurements. J. Acoust. Soc. Am., 115(6):2986-2997, 2004.

[4] K-T. Chen. Study of acoustic transmission through apertures in a wall. Appl. Acoust., 46(2):131 - 151, 1995.

[5] P. Davidsson, J. Brunskog, P. Wernberg, G. Sandberg, and P. Hammer. Analysis of sound transmission loss of double-leaf walls in the low-frequency range using the finite element method. J. Building Acoustics, 11(4):239-257, 2004.

[6] A. Dijckmans, G. Vermeir, and W. Lauriks. Sound transmission through finite lightweight multilayered structures with thin air layers. J. Acoust. Soc. Am., 128(6):3513-3524, 2010.

[7] H. Estrada, P. Candelas, A. Uris, F. Belmar, F. J. Garcia de Abajo, and F. Meseguer. Extraordinary sound screening in perforated plates. Phys. Rev. Lett., 101(8), 2008.

[8] L. Gagliardini, J. Roland, and J.L. Guyader. The use of a functional basis to calculate acoustic transmission between rooms. J. Sound Vibr., 145(3):457-478, 1991.

[9] D. Givoli. Recent advances in the DtN FE method. Arch. Comput. Method Eng., 6:71-116, 1999.

[10] M.C. Gomperts. Sound insulation of circular + slit-shaped apertures. Acustica, 14(1):1-16, 1964.

[11] M.C. Gomperts and T. Kihlman. Sound transmission loss of circular and slitshaped apertures in walls. Acustica, 18(3):144-150, 1967.

[12] V. Hongisto. Sound insulation of doors - Part 1: Prediction models for structural and leak transmission. J. Sound Vibr., 230(1):133 - 148, 2000.

[13] V. Hongisto, J. Keränen, and M. Lindgren. Sound insulation of doors Part 2: Comparison between measurement results and predictions. J. Sound Vibr., 230(1):149 - 170, 2000.

[14] C. Hopkins. Sound insulation. Elsevier Ltd., 2007.

[15] J. L. Horner and K. S. Peat. Approximations for the scattered field potential from higher mode transmission in rectangular apertures. J. Acoust. Soc. Am., 119(6):3568-3576, 2006. 
[16] P. Jean and J.F. Rondeau. A simple decoupled modal calculation of sound transmission between volumes. Acta Acust. United Acust., 88(6):924-933, 2002.

[17] H-J. Kang, J-S. Kim, H-S. Kim, and S-R. Kim. Influence of sound leaks on in situ sound insulation performance. Noise Control Eng. J., 49(3):113-119, 2001.

[18] M-J. Kim and J-H. An. Effect of slit-shaped apertures on sound insulation performance of building elements. Noise Control Eng. J., 57(5):515-523, 2009.

[19] W. Kropp, A. Pietrzyk, and T. Kihlman. On the meaning of the sound reduction index at low frequencies. Acta Acustica, 2:379-392, 1994.

[20] F.P. Mechel. The acoustic sealing of holes and slits in walls. J. Sound Vibr., 111(2):297-336, 1986.

[21] P.M.C. Morse and K.U. Ingard. Theoretical Acoustics. Princeton University Press, 1968.

[22] Y. Naka, A.A. Oberai, and B.G. Shinn-Cunningham. Acoustic eigenvalues of rectangular rooms with arbitrary wall impedances using the interval Newton/generalized bisection method. J. Acoust. Soc. Am., 118(6):3662-3671, 2005.

[23] Numerical Algorithms Group, L. Fox, and J.H. Wilkinson. NAG library manual. NAG Central Office, 1976.

[24] H-H. Park and H-J. Eom. Acoustic scattering from a rectangular aperture in a thick hard screen. J. Acoust. Soc. Am., 101(1):595-598, 1997.

[25] I. Pereira, M. Guettler, and S. Merz. Numerical prediction of the transmission loss of leaks in trimmed panels. Acoustics Australia, 38(3):140 -144, 2010.

[26] A.D. Pierce. Acoustics. An introduction to its physical principles and applications. McGraw Hill, New York, 1981.

[27] J. Poblet-Puig and A. Rodríguez-Ferran. The block Gauss-Seidel method in sound transmission problems. J. Comput. Acoust., 1(18):13-30, 2010.

[28] F. Sgard, H. Nelisse, and N. Atalla. On the modeling of the diffuse field sound transmission loss of finite thickness apertures. J. Acoust. Soc. Am., 122(1):302313, 2007.

[29] E. D. Shabalina, N. V. Shirgina, and A. V. Shanin. High-frequency modes in a two-dimensional rectangular room with windows. Acoust. Phys., 56(4):525-536, 2010.

[30] M. Tournour and N. Atalla. Pseudostatic corrections for the forced vibroacoustic response of a structure-cavity system. J. Acoust. Soc. Am., 107(5):2379-2386, 2000 .

[31] N. Trompette, J.L. Barbry, F. Sgard, and H. Nelisse. Sound transmission loss of rectangular and slit-shaped apertures: Experimental results and correlation with a modal model. J. Acoust. Soc. Am., 125(1):31-41, 2009. 
[32] A Uris, J.M. Bravo, V. Gomez-Lozano, I. Guillen, and J. Llinares. Experimental sound insulation performance of double frame partitions with slits. Appl. Acoust., 69(10):918 - 924, 2008.

[33] A Uris, J.M. Bravo, J. Llinares, and H. Estelles. The influence of slits on sound transmission through a lightweight partition. Appl. Acoust., 65(4):421 - 430, 2004.

[34] T.E. Vigran. Building acoustics. Taylor and Francis, London, 2008.

[35] George P. Wilson and Walter W. Soroka. Approximation to the diffraction of sound by a circular aperture in a rigid wall of finite thickness. J. Acoust. Soc. Am., 37(2):286-297, 1965.

[36] Buye Xu and Scott D. Sommerfeldt. A hybrid modal analysis for enclosed sound fields. J. Acoust. Soc. Am., 128(5):2857-2867, 2010. 